\title{
Proofs on Encrypted Values in Bilinear Groups and an Application to Anonymity of Signatures
}

\author{
Georg Fuchsbauer and David Pointcheval \\ École normale supérieure, LIENS - CNRS - INRIA, Paris, France \\ http://www.di.ens.fr/\{ fuchsbau, ^pointche $\}$
}

\begin{abstract}
We give a generic methodology to unlinkably anonymize cryptographic schemes in bilinear groups using the Boneh-Goh-Nissim cryptosystem and NIZK proofs in the line of Groth, Ostrovsky and Sahai. We illustrate our techniques by presenting the first instantiation of anonymous proxy signatures (in the standard model), a recent primitive unifying the functionalities and strong security notions of group and proxy signatures. To construct our scheme, we introduce various efficient NIZK and witness-indistinguishable proofs.
\end{abstract}

\section{Introduction}

One of the major concerns of modern cryptography is anonymity. Group signatures [CvH91] for example allow members to sign on behalf of a group while remaining anonymous. Other concepts to which anonymity is central are hierarchical group signatures [TW05], identity escrow [KP98] and anonymous credentials [Cha85], to mention only a few. The main issue of these concepts is to demonstrate that a user is entitled to perform a certain task, while not revealing anything about his identity. Zero-knowledge proofs provide the means to do so: prove something without leaking any further information. In particular, non-interactive zero-knowledge (NIZK) proofs [BFM88] have enjoyed numerous applications to achieve anonymity.

Substantial progress has been made in recent years in making NIZK proofs efficient and thus applicable to practical schemes: Groth et al. [GOS06b] show how to efficiently non-interactively prove that a BGNciphertext [BGN05] (cf. Sect. 2) encrypts 0 or 1. Although conceived for purely theoretical purposes, their techniques were used by Boyen and Waters in [BW06] to construct compact group signatures, which they improve in [BW07].

In a different line of research — which has been unified with the one based on BGN in [GS08] —, Groth et al. [GOS06a] based NIZK proofs on a commitment scheme building on linear encryption [BBS04]. The latter is an extension of ElGamal encryption to bilinear groups ${ }^{1}$ and is semantically secure under the decisional linear assumption (DLIN). Keys for GOS-commitments are basically linear encryptions of either 0 or 1 , with the encrypted value determining whether the resulting commitments are perfectly hiding or perfectly binding. Since both types of keys are indistinguishable by DLIN, they inherit a computational version of the other's property from one another.

This scheme has given rise to a multitude of practical NIZK proof systems (see e.g. the full version of [Gro06] for an impressive demonstration of its power), practical implementations of fully-secure group signatures [Gro07] without random oracles [BR93], as well as the introduction of new primitives such as non-interactive anonymous credentials in [BCKL08].

Our Contributions. All the above analyses required ad-hoc security proofs. When extending anonymity to more complex protocols, these proofs quickly become too intricate - unless one manages to provide a generic way to anonymize a large class of proofs. Such a generic anonymization is our first contribution; we generalize the ideas of [BW06,BW07] to BGN-encrypt proofs (and in particular signatures) and prove validity of the encrypted values, for the following category of schemes: the relations checked by the verification algorithm are equations consisting exclusively of products of pairings. (Actually, this is the

\footnotetext{
${ }^{1}$ The decisional Diffie-Hellman assumption (DDH), on which ElGamal relies, does not hold in symmetric bilinear groups.
} 
case for most signature schemes in bilinear groups such as Boneh-Boyen's short signatures [BB04] or Waters' scheme [Wat05].)

We give a methodology to construct proofs demonstrating that encrypted values satisfy certain relations, and show that these proofs do not leak information on the plaintexts, nor additional relations about the plaintexts - providing thus anonymity (unlinkability and untraceability). Moreover, given a set of ciphertexts and a corresponding proof, then without knowledge of the plaintexts, one can re-encrypt (or re-randomize) the ciphertexts and adapt the proof to the new encryptions. In particular, re-randomizations of two sets of ciphertexts and proofs are indistinguishable. This yields a generic method to anonymize schemes in an unlinkable way, such as group signatures ("full anonymity" of the schemes in [BW06] and [BW07] is an immediate consequence of our results), fair contract signing [ASW00], or verifiable encryption [BGLS03], as shown in Sect. 3.2. Since we use encryption to achieve anonymity, the decryption key provides a trapdoor to revoke anonymity in case of abuse, as required by primitives such as group signatures.

In order to illustrate our methodology and to demonstrate its power, our second contribution is the first concrete implementation of anonymous proxy signatures in the standard model. This primitive was recently introduced by Fuchsbauer and Pointcheval [FP08a], who while giving practical applications merely prove theoretical feasibility. It merges group signatures with proxy signatures [MUO96], generalizing the strong security notions of both (in particular, [BMW03,BSZ05] for group signatures and [BPW03] for proxy signatures). Proxy signatures allow consecutive delegation of signing rights while publicly providing the identities of the delegators and the signer with the signed document. Anonymous proxy signatures require that these identities remain hidden: nobody can tell who actually signed or re-delegated, but still anyone can verify that the proxy signer was indeed entitled (via a chain of delegations) to do so. Traceability, i.e. the fact that an authority can revoke anonymity, deters from misuse.

We slightly simplify the model of [FP08a], in that we consider one general opener (instead of having each user choose his own) and anonymity against adversaries without opening oracles (CPA-anonymity [BBS04], a common notion for practical standard-model group signature schemes). Furthermore, we introduce a maximal number of possible delegations. We emphasize that this variant still directly yields dynamic hierarchical group signatures satisfying non-frameability (i.e., the group manager cannot produce signatures that open to a user), while [BW07] only consider the static and non-hierarchical case where the group manager knows every member's secret key.

Overview. We recall some results from the literature on pairing-based cryptography in Sect. 2 and present our methodology in Sect. 3. Before presenting our full scheme in Sect. 5, we mainly focus on constructing a (non-anonymous) scheme for consecutive signature delegations (Sect. 4) to which our methodology can then readily be applied. Its main building block is a signature scheme secure against existential forgeability under chosen message attacks (EUF-CMA) [GMR88], capable of signing public keys for the scheme itself, and whose verification procedure falls in a certain class. The security of the scheme relies on a new assumption presented in Sect. 4.3. The scheme uses a zero-knowledge proof of knowledge [DP92], which we introduce in Sect. 4.2 and of which we sketch an instantiation in Sect. 6. In order to achieve the strong security notions, we design the proof system to satisfy weak simulation soundness, a relaxation of the concept introduced by Sahai [Sah99].

\section{Preliminaries}

We briefly recapitulate the employed concepts from the literature and refer to the cited works for more details. A (symmetric) bilinear group is a tuple $\left(n, \mathbb{G}, \mathbb{G}_{T}, e(\cdot, \cdot), g\right)$ where $\mathbb{G}$ and $\mathbb{G}_{T}$ are two cyclic groups of order $n$ and $g$ is a generator of $\mathbb{G}$. Furthermore, $e(\cdot, \cdot)$ is a non-degenerate bilinear map $\mathbb{G} \times \mathbb{G} \rightarrow \mathbb{G}_{T}$, i.e. $\forall u, v \in \mathbb{G} \forall a, b \in \mathbb{Z}: e\left(u^{a}, v^{b}\right)=e(u, v)^{a b}$ and $e(g, g)$ is a generator of $\mathbb{G}_{T}$. 
The Subgroup Decision Assumption and BGN-Encryption [BGN05]. Let the group order $|\mathbb{G}|=$ $n=p q$ be a product of two primes $p$ and $q$. The subgroup decision assumption (SD) states that no probabilistic polynomial-time (p.p.t.) adversary not knowing the factorization of $n$ can with non-negligible probability distinguish a random element of $\mathbb{G}$ from a random element of $\mathbb{G}_{q}$, the subgroup of order $q$.

The subgroup decision assumption implies semantic security of the following encryption scheme: The public key is the bilinear group (not revealing the factors of its order) and an element $h \in \mathbb{G}_{q}$. The secret key is $q$, i.e. the factorization of the group order. To encrypt a message $m \in\{0, \ldots, T\}$, with $T<p$, choose $r \leftarrow \mathbb{Z}_{n}$ and compute the ciphertext $C:=g^{m} h^{r}$. Since $h$ is of order $q$, we have $C^{q}=\left(g^{m} h^{r}\right)^{q}=\left(g^{q}\right)^{m}$, so $m$ can be recovered by computing $\log _{g^{q}} C^{q}=m$.

The Decisional Linear Assumption and Linear Encryption [BBS04]. Let $\left(p, \mathbb{G}, \mathbb{G}_{T}, e\right)$ be a bilinear group; let $f, h, g$ be generators of $\mathbb{G}$. We call a triple $\left(c_{1}, c_{2}, c_{3}\right) \in \mathbb{G}^{3}$ linear w.r.t. to the basis $(f, h, g)$ iff there exist $r, s \in \mathbb{Z}_{p}$ such that $c_{1}=f^{r}, c_{2}=h^{s}, c_{3}=g^{r+s}$. The decisional linear assumption (DLIN) states that no p.p.t. adversary can distinguish random linear triples w.r.t. a random basis from random triples; that is, given $\left(g, g^{x}, g^{y}, g^{x r}, g^{y s}\right)$ for random $x, y, r, s$, it is hard to distinguish $g^{r+s}$ from a uniformly random element in $\mathbb{G}$.

Assuming DLIN, the following encryption scheme is secure: Choose a secret key $(x, y) \leftarrow\left(\mathbb{Z}_{p}^{*}\right)^{2}$ and publish $p k:=\left(f:=g^{x}, h:=g^{y}, g\right)$. To encrypt a message $m \in \mathbb{G}$, choose $r, s \leftarrow \mathbb{Z}_{p}$ and compute $\operatorname{Enc}(p k, m ;(r, s)):=\left(f^{r}, h^{s}, m g^{r+s}\right)$. Any $(u, v, w)$ can be decrypted by computing $u^{-x^{-1}} v^{-y^{-1}} w=$ $g^{-r} g^{-s} m g^{r+s}=m$.

GOS-Commitments [GOS06a]. The following homomorphic commitment scheme is based on linear encryption: The commitment key is a public key for linear encryption $(f, h, g)$ and a triple $(u, v, w)$ which is an encryption of either 1 or $g$ (i.e., $\left(f^{r_{u}}, h^{s_{v}}, g^{r_{u}+s_{v}}\right)$ or $\left(f^{r_{u}}, h^{s_{v}}, g^{r_{u}+s_{v}+1}\right)$ for random $r_{u}, s_{v} \in$ $\mathbb{Z}_{p}$ ). The first leads to a perfectly hiding key, while the latter constitutes a perfectly binding key. Now $\operatorname{Com}((f, h, g, u, v, w), m ;(r, s)):=\left(u^{m} f^{r}, v^{m} h^{s}, w^{m} g^{r+s}\right)$ is a commitment to $m \in \mathbb{Z}_{p}$ for random $r, s$. Note that for perfectly hiding keys for any message $m$ this is a random encryption of 0 while in the binding case, it encrypts $g^{m}$.

\section{The Leak-Tightness Lemma}

In [BW07], Boyen and Waters use the following strategy to construct efficient group signatures without random oracles: First, they construct two-level hierarchical signatures (a.k.a. certified signatures) that satisfy unforgeability ("traceability"), such that signatures consist of group elements only and can be verified by checking pairing-product equations (cf. Lemma 1). They then convert the scheme into a group signature scheme, obtaining anonymity by BGN-encrypting the signature components and adding proofs for the plaintexts satisfying the verification equations. Considerable effort is then dedicated to showing that their specific proofs do not leak information on the plaintexts.

In fact, as shown by the following lemma, proofs of this kind generally do not leak any additional information on the encrypted values. Thus, full anonymity of [BW06] and [BW07] follows immediately from the lemma. We first state the - somewhat technical - results and clarify their relevance in the subsequent discussion.

Lemma 1 (Leak tightness). Let $\left(n, \mathbb{G}, \mathbb{G}_{T}, e, g\right)$ be a bilinear group, and let $a_{j}, b_{j} \in \mathbb{G}, \delta_{j, i}, \varepsilon_{j, i} \in \mathbb{Z}_{n}$ for $1 \leq j \leq \ell, 1 \leq i \leq m$. Let $\left(X_{i}\right)_{i=1}^{m} \in \mathbb{G}^{m}$ satisfy a pairing-product equation $E_{\left(a_{j}, b_{j}\right)_{j}}$ that is

$$
E_{\left(a_{j}, b_{j}\right)_{j}}\left(X_{1}, \ldots, X_{m}\right): \quad \prod_{j=1}^{\ell} e\left(a_{j} \prod_{i=1}^{m} X_{i}^{\delta_{j, i}}, b_{j} \prod_{i=1}^{m} X_{i}^{\varepsilon_{j, i}}\right)=\mathbf{1} .
$$


1. Let $H \in \mathbb{G},\left(\rho_{i}\right)_{i=1}^{m} \in \mathbb{Z}_{n}^{m}$. Then $\widetilde{X}_{i}:=X_{i} H^{\rho_{i}}$ for $1 \leq i \leq m$ satisfy

$$
\prod_{j} e\left(a_{j} \prod_{i} \widetilde{X}_{i}^{\delta_{j, i}}, b_{j} \prod_{i} \widetilde{X}_{i}^{\varepsilon_{j, i}}\right)=e\left(H, P_{E}\left(\left(X_{i}\right),\left(\rho_{i}\right)\right)\right)
$$

where $P_{E}\left(\left(X_{i}\right),\left(\rho_{i}\right)\right):=\prod_{j}\left(\left(a_{j} \prod_{i} X_{i}^{\delta_{j, i}}\right)^{\sum \varepsilon_{j, i} \rho_{i}}\left(b_{j} \prod_{i} X_{i}^{\varepsilon_{j, i}}\right)^{\sum \delta_{j, i} \rho_{i}} H^{\left(\sum \delta_{j, i} \rho_{i}\right)\left(\sum \varepsilon_{j, i} \rho_{i}\right)}\right)$.

2. Given $\left(X_{i}\right)$ and $\left(X_{i}^{\prime}\right)$ both satisfying $E$, and $\left(\rho_{i}\right)$, $\left(\rho_{i}^{\prime}\right)$, s.t. for all $1 \leq i \leq m: X_{i} H^{\rho_{i}}=X_{i}^{\prime} H^{\rho_{i}^{\prime}}$, then

$$
P_{E}\left(\left(X_{i}\right),\left(\rho_{i}\right)\right)=P_{E}\left(\left(X_{i}^{\prime}\right),\left(\rho_{i}^{\prime}\right)\right) .
$$

3. Let $|G|=p q$, let $a_{j}, b_{j}, X_{i} \in \mathbb{G}_{p} ; c_{j}, d_{j}, Y_{i} \in \mathbb{G}_{q}$ for all $i, j$. If $\left(X_{i}\right)$ satisfy $E_{\left(a_{j}, b_{j}\right)_{j}}$ and $\left(Y_{i}\right)$ satisfy $E_{\left(c_{j}, d_{j}\right)_{j}}$, then $\left(X_{i} Y_{i}\right)$ satisfy $E_{\left(a_{j} c_{j}, b_{j} d_{j}\right)_{j}}$.

4. Let furthermore $H \in \mathbb{G}_{q}$ and $\theta \in \mathbb{N}$ be such that $\theta \equiv 1(\bmod p)$ and $\theta \equiv 0(\bmod q)$. If $\left(\widetilde{X}_{i}\right) \in \mathbb{G}$ satisfy $\tilde{E}_{\left(a_{j} c_{j}, b_{j} d_{j}\right)_{j}}$ for some $P_{E}$, then $\left(\tilde{X}_{i}^{\theta}\right)$ satisfy $E_{\left(a_{j}, b_{j}\right)_{j}}$.

See Appendix D.1 for the proof. We give a brief description of the lemma's content: Let $\left(X_{i}\right)$ be a vector of group elements satisfying relation $E$; think of the $X_{i}$ 's as components of a digital signature and $E$ being the verification relation. If $H \in \mathbb{G}_{q}$ then $\widetilde{X}_{i}$ as defined in (1) is a BGN-encryption of $X_{i}$ using randomness $\rho_{i}$. Given $\left(\widetilde{X}_{i}\right)$, the element $P_{E}$ can be seen as a proof that the plaintexts in $\left(\widetilde{X}_{i}\right)$ satisfy $E$, which is verified by checking $\widetilde{E}$.

While (1) states that every proof constructed as described passes verification, (4) ensures soundness: if there exists a $P_{E}$ such that $\left(\widetilde{X}_{i}\right)$ and $P_{E}$ satisfy $\widetilde{E}$ in $\mathbb{G}$, then their projections $\left(\widetilde{X}_{i}^{\theta}\right)$ into $\mathbb{G}_{p}$ satisfy $E$ in $\mathbb{G}_{p}$. We will use this fact to reduce a forgery in an "anonymized" scheme in $\mathbb{G}$ to a forgery in an underlying scheme in $\mathbb{G}_{p}$; in [BW06] for example a forged group signature is translated to a forgery of a certified signature this way.

If we have equations $E_{\left(a_{j}, b_{j}\right)_{j}}$ in $\mathbb{G}_{p}$ and $E_{\left(c_{j}, d_{j}\right)_{j}}$ in $\mathbb{G}_{q}$, and values $\left(X_{i}\right),\left(Y_{i}\right)$ satisfying them respectively, then their products satisfy equation $E_{\left(a_{j} c_{j}, b_{j} d_{j}\right)_{j}}$ in $\mathbb{G}$ due to (3), which we will be useful in our simulations.

Now the main result is (2): Assume $H \in \mathbb{G}$, rather than in $\mathbb{G}_{q}$, which is indistinguishable by the subgroup decision (SD) assumption. In this case each $\widetilde{X}_{i}$ is perfectly random: Given an "encryption" $\widetilde{X}_{i}$, then for any potential plaintext $X_{i}$, there exists randomness $\rho_{i}:=\log _{H}\left(\widetilde{X}_{i} / X_{i}\right)$ leading to $\widetilde{X}_{i}$. Now, $(2)$ states that given $\left(\widetilde{X}_{i}\right)$, any vector of such pairs of plaintexts/randomness $\left(X_{i}, \rho_{i}\right)_{i=1}^{m}$ "explaining" $\left(\widetilde{X}_{i}\right)$ leads to exactly the same proof $P_{E}$, which means that the proof leaks no information on the plaintext.

Remark 1 (Unlinkably re-randomizing randomized values). Consider a vector $\left(X_{i}\right)$ satisfying $E$, but with right-hand side $e\left(H, P^{\prime}\right)$ instead of 1 . Again, let $\widetilde{X}_{i}:=X_{i} H^{\rho_{i}}$ for all $i$. Then $\left(\widetilde{X}_{i}\right)$ satisfies $\widetilde{E}$ with $e\left(H, P^{\prime} \cdot P_{E}\left(\left(X_{i}\right),\left(\rho_{i}\right)\right)\right)$ as right-hand side. So, given a proof $P$ for randomized $\left(\widetilde{X}_{i}\right)$ satisfying $\widetilde{E}$, one can re-randomize the $\left(\widetilde{X}_{i}\right)$ using fresh $\rho_{i}^{\prime}$ and adapt the proof (without knowledge of the plaintexts!) by setting $P_{\text {new }}:=P \cdot P_{E}\left((\widetilde{X}),\left(\rho_{i}^{\prime}\right)\right)$. If $\left(\left(\widetilde{X}_{i}\right), P\right)$ and $\left(\left(\widetilde{Y}_{i}\right), P^{\prime}\right)$ both satisfy $\widetilde{E}$, then their re-randomizations are indistinguishable by SD and Lemma $1(2)$.

\subsection{The Waters Signature Scheme}

We review the scheme from [Wat05] to sign messages $M=\left(M_{1}, \ldots, M_{m}\right) \in\{0,1\}^{m}$, which will be used several times in the remainder of the paper.

Setup. Choose a bilinear group $\left(n, \mathbb{G}, \mathbb{G}_{T}, e, g\right)$. The parameters are $g_{2} \leftarrow \mathbb{G}^{*}$ and a vector $\boldsymbol{u}:=\left(u_{0}, u_{1}, \ldots, u_{m}\right) \leftarrow$ $\mathbb{G}^{m+1}$. Choose a secret key $x \leftarrow \mathbb{Z}_{m}$, and define the public key as $X:=g^{x}$.

For convenience, we define the following function $\mathcal{F}(M):=\prod_{i=1}^{m} u_{i}^{M_{i}}$. 
Signing. Choose $r \leftarrow \mathbb{Z}_{p}$ and define the signature as $\sigma:=\left(g_{2}^{x}\left(u_{0} \mathcal{F}(M)\right)^{r}, g^{-r}\right)$.

Verification. A signature $\sigma=\left(\sigma_{1}, \sigma_{2}\right)$ is accepted for a message $M$ iff

$$
e\left(\sigma_{1}, g\right) e\left(u_{0} \mathcal{F}(M), \sigma_{2}\right)=e\left(g_{2}, X\right) .
$$

Security. EUF-CMA follows from hardness of the computational Diffie-Hellman assumption (CDH) in the underlying group.

\subsection{Applying Lemma 1 to Construct Verifiable Encryption}

To exemplify our techniques, we construct a verifiable-encryption scheme in the standard model, which we only sketch due to space limitations. Suppose, we want to encrypt a signature and prove that the plaintext satisfies the signature verification relation. Lemma 1 lets us do so if the verification procedure consists merely of verifying pairing-product equations, as is the case for Waters' scheme. Moreover, if the signatures are EUF-CMA then a similar property holds for encryption/proof pairs: Even after querying such pairs for messages of its choice, no adversary can produce a valid pair for a new message.

We construct a scheme $\mathcal{E S}$ for encrypted signatures: Given a plain signature in scheme $\mathcal{S}$, independently BGN-encrypt all its components and add a proof $P_{E}$ for each verification equation $E$, as defined in Lemma 1(1). Indistinguishability of the hidden elements follows from the SD assumption combined with (2): Replacing $H \in \mathbb{G}_{q}$ by a random element from the entire group $\mathbb{G}$ is indistinguishable by SD. Now the encryptions are perfectly random and the proofs do not reveal any information either; every hypothesis $\left(X_{i}\right)$ on the plaintexts of $\left(\widetilde{X}_{i}\right)$ leads to the same proof.

Unforgeability of $\mathcal{E S}$ is inherited from scheme $\mathcal{S}$ defined in subgroup $\mathbb{G}_{p}$ : Lemma $1(3)$ allows us to simulate all oracle queries and (4) lets us transform a forgery in $\mathcal{E S}$ to a forgery in $\mathcal{S}$; more precisely: Given an adversary $\mathcal{A}$ against $\mathcal{E S}$ in $\mathbb{G}$, we construct $\mathcal{B}$ against $\mathcal{S}$ in $\mathbb{G}_{p}$ as follows: After receiving the parameters of $\mathcal{S}, \mathcal{B}$ produces parameters and the public key for a twin instance $\mathcal{T S}$ of $\mathcal{S}$, but in subgroup $\mathbb{G}_{q}$ (knowing thus the secret key). Then $\mathcal{B}$ constructs scheme $\mathcal{E S}$ in $\mathbb{G}$ whose parameters are the products of those of $\mathcal{S}$ and $\mathcal{T S}$.

Whenever $\mathcal{A}$ performs an oracle query, $\mathcal{B}$ splits all involved group elements (if any) into their components in $\mathbb{G}_{p}$ (by raising them to the $\theta$-th power as in (4)) and their components in $\mathbb{G}_{q}$ by raising them to the power of $\theta_{q}$, with $\theta_{q} \equiv 0(\bmod p)$ and $\theta_{q} \equiv 1(\bmod q)$. The $p$-parts are submitted to $\mathcal{B}$ 's own oracle, while the action on the $q$-parts can be performed by $\mathcal{B}$ itself. The two results are then combined to a solution in $\mathbb{G}$ by multiplying them component-wise. (3) guarantees validity as the products satisfy the equations in group $\mathbb{G}$ when both components satisfy the equations in their respective subgroups. Finally, a forgery returned by $\mathcal{A}$ can be translated to one for $\mathcal{S}$, again via (4), giving $\mathcal{B}$ the same success probability as $\mathcal{A}$.

To further illustrate our methodology, we give an instantiation of "anonymous proxy signatures". We first construct a (non-anonymous) delegation scheme whose verification relations satisfy the requirements of Lemma 1. To instantiate the generic concept of such a scheme, the most important tool is the following: a Lemma-1-compatible EUF-CMA-secure signature scheme, where the messages to be signed are vectors of public keys of the scheme itself. ${ }^{2}$ This is the main difference to previous certified-signature schemes (on which group signatures build), where the certification and the signature itself are not based on the same mechanism, excluding thus consecutive delegation. In order to motivate our proceeding we briefly review the notions from [FP08a] in the next section.

\footnotetext{
${ }^{2}$ Note that we cannot simply hash the vector of messages and sign the hash value, as we will later encrypt the messages and prove that the signature is valid on the plaintexts.
} 


\subsection{Definition and Security of Anonymous Proxy Signatures}

In an anonymous proxy signature scheme, there are the following protagonists: The issuer enrolls users in the system, the users can delegate and sign on behalf of other users, and the opener is able to trace the hidden delegators and the signer from a proxy signature in case of misuse.

The scheme consists of 7 algorithms: Setup produces the public parameters, the issuer's secret key and the opening key. Algorithm UKGen is run by the users in order to produce a key pair, the public key of which is registered by the issuer running Enroll. A user can delegate her signing rights by producing a warrant with DIg taking as input her secret key and the delegatee's public key. DIg also provides the possibility to re-delegate when given a warrant as additional argument. Now using a warrant, users can "proxy sign" messages running PSig, whereas the resulting signatures are verifiable via PVer using the first ("original") delegator's public key only. Algorithm Open allows the opener holding the opening key to reveal the delegators and the signer.

We overview the required security notions and refer to the full version or [FP08a] for the rigorous definitions:

Anonymity. The experiment for anonymity is the following: Consider an adversary getting the issuer's key and who in a first phase returns an original delegator's public key, two pairs consisting of a warrant and a secret key each, and a message. Now, flip a random bit and depending on the outcome give the adversary a signature produced using either the first or the second warrant/secret-key pair. Then as long as both warrants result from the same number of delegations and both lead to valid signatures, the adversary cannot decide the value of the flipped bit with probability more than a half.

Traceability. No adversary, after enrolling arbitrarily many users via an Enroll-oracle, can produce a signature which cannot be opened. Thus, every valid signature can be traced to registered users.

Non-Frameability. No adversary, even when colluding with the issuer and the opener, can frame honest users. More precisely, give the adversary all keys returned by Setup, and oracles to create honest users and ask delegations and signatures of them-or adaptively corrupt them by asking their secret key. Then the adversary is not able to produce a valid signature whose opening yields an honest user for a delegation or a signing he has not been queried for.

Remark 2. Remark 1 hints that our scheme actually achieves a stronger notion of anonymity where even to a delegatee the preceding delegators are anonymous.

\section{A Consecutive Signature-Delegation Scheme}

\subsection{Overview}

A Generic Construction. The issuer and each user create a key pair for an EUF-CMA-secure signature scheme. To enroll a user, the issuer signs her public key, creating thus a certificate sent to the user. If user $U_{1}$ wants to delegate $U_{2}$, she sends him a signature on her own and $U_{2}$ 's public key, called warrant. To re-delegate to $U_{3}, U_{2}$ sends her his certificate cert $_{2}$ received from the issuer, the warrant warr $\operatorname{wa}_{1 \rightarrow 2}$ received from $U_{1}$, and warr $_{1 \rightarrow 2 \rightarrow 3}$, a signature on $\left(p k_{1}, p k_{2}, p k_{3}\right)$, the user's public keys. Now to sign a message $M$ on behalf of $U_{1}, U_{3}$ produces a signature $\sigma$ on $\left(p k_{1}, p k_{2}, p k_{3}, M\right)$. The (non-anonymous) proxy signature is $\Sigma:=\left(\right.$ warr $_{1 \rightarrow 2}$, pk $_{2}$, cert $_{2}$, warr $_{1 \rightarrow 2 \rightarrow 3}$, pk $\left._{3}, \operatorname{cert}_{3}, \sigma\right)$.

Remark 3 (Delegating for specific tasks only). The scheme can easily be extended, so that delegation of signing rights can be done for specific tasks only — as proposed by [FP08a] — as follows: When delegating, sign $\left(p k_{1}, \ldots, p k_{i}\right.$, task) rather than the public keys only; likewise for proxy signing. The verification procedure takes the task tag as additional argument and the verification relations are adapted respectively. 
Instantiation. We instantiate the generic scheme by choosing Waters' signature scheme (cf. Sect. 3.1) as EUF-CMA-secure scheme, which supports the hierarchical nature of the messages to be signed. Unfortunately, at the same time, this limits us to a fixed maximal number of delegations.

The messages in the Waters scheme are bit-strings, while we need to sign vectors of public keys (i.e., group elements) for the scheme itself. We solve this shortcoming as follows: Instead of signing public keys, we sign the bits of the private keys - which the signer should obviously not learn. We take thus advantage of the fact that Waters signatures can be computed and verified without knowledge of the message if its hash value $F=\mathcal{F}(M)=\prod_{i=1}^{m} u_{i}^{M_{i}}$ is given instead. On the other hand, the assumption we introduce in Sect. 4.3 implies that the hash value hides enough information about the secret key. In particular, it states that the public key and the secret key's hash look unrelated.

The private key's hash value can be precomputed by its owner and then be used directly by the delegator to produce a signature. We define thus the following two functions: ${ }^{3}$

$$
\begin{aligned}
& \operatorname{FSig}(x, F):=\left(\bar{g}^{x}(\bar{u} F)^{r}, g^{-r}\right) \text { for random } r \leftarrow \mathbb{Z}_{p}, \\
& \operatorname{FVer}\left(X, F,\left(\sigma_{1}, \sigma_{2}\right)\right)=1 \text { iff } e\left(\sigma_{1}, g\right) e\left(\bar{u} F, \sigma_{2}\right)=e(\bar{g}, X) .
\end{aligned}
$$

Now we need to add a NIZK proof of consistency of the hash with the corresponding public key, which we discuss in the next section. Anticipating, we note that the secret key must be extractable from such a proof, so we can reduce unforgeability of delegations (i.e., non-frameability) of our scheme to security of Waters signatures. We emphasize the fact that verifying the NIZK proof must exclusively consist of checking pairing-product equations to be compatible with the Leak-Tightness Lemma.

\subsection{ZK Proof of Equality of Logarithm and Hash Preimage}

As mentioned above, in order to prove consistency of a public key $X=g^{x}$ with the hash value of its private key $F=\mathcal{F}(x)$, in Sect. 6 we construct a zero-knowledge proof system $\Pi_{\mathrm{X} \leftrightarrow \mathrm{F}}$ for NP-relation

$$
R_{\mathrm{X} \leftrightarrow \mathrm{F}}:=\left\{((X, F), x) \mid X=g^{x}, F=\mathcal{F}(x)\right\} .
$$

The NP-language $\mathcal{L}_{\mathrm{X} \leftrightarrow \mathrm{F}}$ defined by it is then indistinguishable from $\mathbb{G}^{2}$ by the XF-assumption given in the next section. We require $\Pi_{\mathrm{X} \leftrightarrow \mathrm{F}}$ to have the following properties:

- Verification of a proof consists of checking pairing-product equations.

- The proof is a proof of knowledge at the same time, i.e., we can extract witness $x$. Furthermore, extraction must be efficient and consequently cannot rely on rewinding techniques.

- We can simulate proofs for any (possibly false) statements $\left(g^{x_{1}}, \mathcal{F}\left(x_{2}\right)\right)$ without knowledge of $\left(x_{1}, x_{2}\right)$.

- Even after seeing a simulated proof of a random (not necessarily true) statement, no adversary can produce a proof for a false statement; in addition, from every valid proof, the witness can still be extracted. This property, defined below, is a relaxation of the standard notion of simulation soundness where it is the adversary that chooses the statement to be simulated.

A NIZK proof of knowledge is a tuple $\left(\mathrm{K}, \mathrm{P}, \mathrm{V}, \mathrm{Sim}_{1}, \mathrm{Sim}_{2}\right.$, Ext), where $\mathrm{K}$ generates the common reference string (CRS) crs and $\mathrm{P}$ produces proofs that are verified via V. Simulator Sim $_{1}$ outputs a crs, a trapdoor tr which allows $\mathrm{Sim}_{2}$ to simulate proofs, and an extraction key ek, used by Ext to extract the witness.

Definition 2. A proof of knowledge $\Pi=\left(\mathrm{K}, \mathrm{P}, \mathrm{V}, \operatorname{Sim}_{1}, \operatorname{Sim}_{2}\right.$, Ext $)$ for NP-language $\mathcal{L}$ is weakly simulation sound if for every p.p.t. $\mathcal{A}$ the following probability is negligible in the security parameter $\lambda$ :

$$
\begin{aligned}
\operatorname{Pr}\left[(\operatorname{crs}, t r, e k) \leftarrow \operatorname{Sim}_{1}\left(1^{\lambda}\right) ; y\right. & \leftarrow \mathcal{L} \cup \overline{\mathcal{L}} ; \pi \leftarrow \operatorname{Sim}_{2}(\operatorname{tr}, y) ;\left(y^{*}, \pi^{*}\right) \leftarrow \mathcal{A}(\operatorname{crs},(y, \pi)) ; \\
& \left.w^{*} \leftarrow \operatorname{Ext}\left(e k,\left(y^{*}, \pi^{*}\right)\right): y^{*} \neq y \wedge\left(y^{*}, w^{*}\right) \notin R_{\mathcal{L}} \wedge \vee\left(c r s, y^{*}, \pi^{*}\right)=1\right]
\end{aligned}
$$

\footnotetext{
${ }^{3}$ Note that FSig, FVer do not constitute a secure signature scheme on their own; a successful forgery must include the message's bits $\left(M_{i}\right)_{i=1}^{m}$ s.t. $F=\mathcal{F}\left(\ldots, M_{i}, \ldots\right)$ in order to be reducible to CDH.
} 
Weak simulation soundness (WSS) is implied by the following strengthening of zero-knowledge, where the adversary trying to distinguish between a real and a simulated proof is now provided with an extraction oracle.

Definition 3. A proof of knowledge $\Pi=\left(\mathrm{K}, \mathrm{P}, \mathrm{V}, \mathrm{Sim}_{1}\right.$, $\operatorname{Sim}_{2}$, Ext $)$ is extraction zero knowledge if for every p.p.t. adversary $\mathcal{A}=\left(\mathcal{A}_{1}, \mathcal{A}_{2}\right)$ we have:

$$
\left|\operatorname{Pr}\left[\operatorname{Exp}_{\Pi,, \mathcal{A}}^{\mathrm{zk}}(\lambda)=1\right]-\operatorname{Pr}\left[\operatorname{Exp}_{\Pi, \mathcal{A}}^{\mathrm{zk}-\mathrm{S}}(\lambda)=1\right]\right|=\operatorname{negl}(\lambda),
$$

with

$$
\begin{aligned}
& \operatorname{Exp}_{\Pi, \mathcal{A}}^{\mathrm{zk}}(\lambda) \\
& \quad(c r s, e k) \leftarrow \mathrm{K}\left(1^{\lambda}\right) \\
& \quad(y, w, s t) \leftarrow \mathcal{A}_{1}(c r s: \operatorname{Ext}(e k, \cdot, \cdot)) \\
& \quad \pi \leftarrow \mathrm{P}(\operatorname{crs}, y, w) \\
& \quad b \leftarrow \mathcal{A}_{2}(s t, \pi: \operatorname{Ext}(e k, \cdot, \cdot))
\end{aligned}
$$

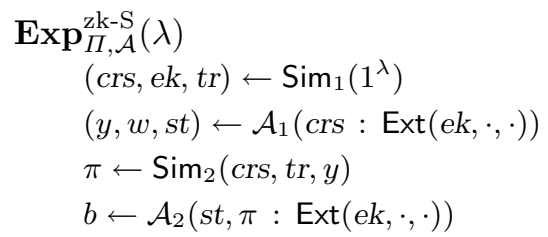

Claim 1 (EZK implies WSS). Let $\mathcal{L}$ be a language which no p.p.t. adversary can decide with non-negligible probability; let $\Pi$ be an extraction-zero-knowledge proof of knowledge for $\mathcal{L}$. Then $\Pi$ is weakly simulation sound.

Proof. Consider the following game:

$$
\begin{aligned}
\text { GAme } 0 & (\text { crs, ek }) \leftarrow \mathrm{K}\left(1^{\lambda}\right) ;(y, w) \leftarrow R_{\mathcal{L}} ; \pi \leftarrow \mathrm{P}(\operatorname{crs}, y, w) ; \\
& \left(y^{*}, \pi^{*}\right) \leftarrow \mathcal{A}(\operatorname{crs},(y, \pi)) ; w^{*} \leftarrow \operatorname{Ext}\left(e k,\left(y^{*}, \pi^{*}\right)\right) ; \\
& \text { return } 1 \text { iff } y^{*} \neq y \wedge\left(y^{*}, w^{*}\right) \notin R_{\mathcal{L}} \wedge \mathrm{V}\left(\text { crs, } y^{*}, \pi^{*}\right)=1
\end{aligned}
$$

Soundness of $\Pi$ implies that $\mathcal{A}$ can win Game 0 with at most negligible probability. Now define Game 1 replacing $\mathrm{K}$ and $\mathrm{P}$ by $\operatorname{Sim}_{1}$ and $\operatorname{Sim}_{2}$, respectively. Games 0 and 1 are indistinguishable by EZK, since a distinguisher can perfectly simulate the games because of its extraction oracle. Finally, a distinguisher between Game 1 and the wss game would contradict the assumption on $\mathcal{L}$ (neither game uses the witness $w)$.

\subsection{The XF-Assumption}

The XF-assumption basically states that for someone seeing a public key $X=g^{x}$ without knowing the secret key $x$, the hash $\mathcal{F}(x)$ of the latter looks random. We will utilize this when reducing non-frameability of our delegation scheme to unforgeability of Waters signatures, where we will have to produce hashes corresponding to unknown secret keys. Proof system $\Pi_{\mathrm{X} \leftrightarrow \mathrm{F}}$ allows us to simulate the consistency proofs, but however, replacing an element of $\mathcal{L}_{\mathrm{X} \leftrightarrow \mathrm{F}}$ by one outside the language must be indistinguishable to guarantee simulation.

Moreover, having to simulate hash values for all delegation levels (cf. Sect. 4.4 for the details), we will generalize our assumption: Given $X=g^{x_{0}}$ and $\Lambda$ hash values $F_{i}=\mathcal{F}_{i}\left(x_{i}\right)$, for different hash functions $\mathcal{F}_{i}$, it is hard to tell whether all $x_{i}$ 's are equal. Intuitively, the assumption states that values $F_{i}$ do not reveal more information about $x$ than $X$.

Definition 4. Let $\Lambda, m \in \mathbb{N},\left(n, \mathbb{G}, \mathbb{G}_{T}, e, g\right) \leftarrow \mathcal{G}\left(1^{\lambda}\right)$ be a bilinear group, let $\left(\left(u_{i, j}\right)_{j=1}^{m}\right)_{i=1}^{\Lambda} \in \mathbb{G}^{\Lambda \times m}$. We define the $i^{\text {th }}$ hash of $\left(x_{1}, \ldots, x_{m}\right) \in\{0,1\}^{m}$ :

$$
\mathcal{F}_{i}\left(x_{1}, \ldots, x_{m}\right):=\prod_{j=1}^{m} u_{i, j}^{x_{j}}
$$


We say the $(\Lambda, m)$-XF-Assumption holds for $\mathcal{G}$ if it is difficult to distinguish the NP-language

$$
\mathcal{L}_{\mathrm{X} \leftrightarrow \mathrm{F}}:=\left\{\left(X,\left(F_{i}\right)_{i=1}^{\Lambda}\right) \in \mathbb{G}^{\Lambda+1} \mid \exists x:=\left(x_{1}, \ldots, x_{m}\right) \in\{0,1\}^{m}: X=g^{\sum x_{i} 2^{i-1}} \wedge \bigwedge_{i=1}^{\Lambda} F_{i}=\mathcal{F}_{i}(x)\right\}
$$

from $\mathbb{G}^{\Lambda+1}$, that is, for all p.p.t. adversaries $\mathcal{A}$, the following function is negligible in $\lambda$ :

$$
\begin{aligned}
\mid \operatorname{Pr}\left[\left(n, \mathbb{G}, \mathbb{G}_{T}, e, g\right) \leftarrow \mathcal{G}\left(1^{\lambda}\right) ; \boldsymbol{u} \leftarrow \mathbb{G}^{\Lambda \times m} ; x \leftarrow\{0,1\}^{m}:\right. & \\
& \left.\mathcal{A}\left(n, \mathbb{G}, \mathbb{G}_{T}, e, g, \boldsymbol{u}, g^{\sum x_{i} 2^{2-1}}, \prod u_{1, i}^{x_{i}}, \ldots, \prod u_{\Lambda, i}^{x_{i}}\right)=1\right] \\
-\operatorname{Pr}\left[\left(n, \mathbb{G}, \mathbb{G}_{T}, e, g\right) \leftarrow \mathcal{G}\left(1^{\lambda}\right) ; \boldsymbol{u} \leftarrow \mathbb{G}^{\Lambda \times m} ; X, F_{1}, \ldots, F_{\Lambda} \leftarrow \mathbb{G}:\right. & \left.\mathcal{A}\left(n, \mathbb{G}, \mathbb{G}_{T}, e, g, \boldsymbol{u}, X, F_{1}, \ldots, F_{\Lambda}\right)=1\right]
\end{aligned}
$$

Note that the assumption satisfies Naor's falsifiability criterion [Nao03]. We give some more intuition on the assumption.

Comparison to DDH and DLIN. Consider the $(1, m)$-XF-Assumption in a group $\mathbb{G}$ with $2^{\lambda-1} \leq$ $|\mathbb{G}|<2^{\lambda}$, and $m=\lambda-1$ : Given $\left(g, u_{1}, \ldots, u_{m}, X, F\right)$, decide whether there exist $x_{i} \in \mathcal{S}:=\{0,1\}$, s.t. $X=g^{\sum x_{i} 2^{i-1}}$ and $F=\prod u_{i}^{x_{i}}$.

If we set $m=1$ and $\mathcal{S}=\mathbb{Z}_{2^{\lambda}}$, we get DDH-which is easy in bilinear groups. However, case $m=2$, $\mathcal{S}=\mathbb{Z}_{2^{\lambda / 2}}$ (i.e., $X=g^{x_{1}+x_{2} 2^{\lambda / 2}} \stackrel{?}{\Rightarrow} F=u_{1}^{x_{1}} u_{2}^{x_{2}}$ ) can already be considered hard, since it is implied by a variant of DLIN, where $r, s$ are randomly chosen from a smaller set $\mathcal{S}$ : An instance $\left(Y=g^{y}, Z=\right.$ $\left.g^{z}, R=g^{y r}, S=g^{z s}, T \in\left\{g^{r+s}, g^{t}\right\}\right)$ of DLIN with $r, s \in \mathcal{S}$ can be decided by running the XF-decider on $\left(u_{1}=Y, u_{2}=Z, X=T, F=R \cdot S^{2^{\lambda / 2}}\right)$.

Now, if we continue the process of increasing $m$ while at the same time reducing the set of possible values for $x_{i}$, we end up with the XF-assumption.

Relation to the DL Problem with Auxiliary Information. Consider the problem of computing $x=\log X$ on input $(X, F) \in \mathcal{L}_{\boldsymbol{u}}$, i.e., in addition to instance $X$, a hash value $F=\mathcal{F}_{\boldsymbol{u}}(x):=\prod u_{i}^{x_{i}}$ of the logarithm is given. Suppose, there exists an algorithm $\mathcal{A}$ that on input $(\boldsymbol{u}, X, F)$ decides whether $F=\prod u_{i}^{x_{i}}$ for $x:=\log X$, thus breaking the XF-assumption. Then we can construct an algorithm $\mathcal{B}$ that given $(X, F) \in \mathcal{L}_{\boldsymbol{u}}$ computes $x=\log X$ : For $1 \leq i \leq m$, choose random $u_{i}^{*}$ and run $\mathcal{A}$ on $\left(U_{i}:=\right.$ $\left.\left(u_{1}, \ldots, u_{i-1}, u_{i}^{*}, u_{i+1}, \ldots, u_{m}\right), X, F\right)$. If $x_{i}=0$, then $(X, F) \in \mathcal{L}_{U_{i}}$, whereas this is only the case with negligible probability if $x_{i}=1 . \mathcal{B}$ can thus extract $x$ bit-by-bit.

\subsection{Implementation of the Delegation Scheme $\mathcal{D S}$}

Based on the ideas from Sect. 4.1, we give implementations of the algorithms introduced in Sect. 3.3 (where $\lambda$ is the security parameter and $\Lambda-1$ is the maximum delegation "depth", that is, the number of possible delegations from the original delegator to the proxy signer).

$\operatorname{Setup}\left(1^{\lambda}, \Lambda\right) \quad-$ Choose a bilinear group $g P a r:=\left(p, \mathbb{G}, \mathbb{G}_{T}, e, g\right) \leftarrow \mathcal{G}\left(1^{\lambda}\right)$.

- Define $m$, the maximal length of messages to be signed, as $m:=\lambda-1$.

- Choose Waters parameters to sign messages consisting of $\Lambda \cdot m$ bits: $s$ Par $:=\left(\bar{g}, \bar{u},\left(u_{i, 1}, \ldots, u_{i, m}\right)_{i=1}^{\Lambda}\right) \leftarrow \mathbb{G}^{\Lambda m+2}$

- For $1 \leq i \leq \Lambda$, choose $\mathrm{crs}_{i}$, a common reference string for $\Pi_{\mathrm{X} \leftrightarrow \mathrm{F}}$ for parameters $\left(u_{i, j}\right)_{j=1}^{m}$.

The issuer chooses an issuing key ik $:=\omega \leftarrow \mathbb{Z}_{p}$ and defines $\Omega:=g^{\omega}$. The public parameters are

$$
p p:=\left(g P a r, s P a r,\left(\operatorname{crs}_{i}\right)_{i=1}^{\Lambda}, \Omega\right) \text {. }
$$


UKGen $(p p)$ Choose a random $x \leftarrow \mathbb{Z}_{2^{m}}$ and set $X:=g^{x}$. Define the public key $p k:=\left(X,\left(F_{i}, P_{i}\right)_{i=1}^{\Lambda}\right)$, where $F_{i}:=\mathcal{F}_{i}(x)$, is the $i^{\text {th }}$ hash (cf. Def. 4) and $P_{i}:=\mathrm{P}_{\mathrm{X} \leftrightarrow \mathrm{F}}\left(\operatorname{crs}_{i},\left(X, F_{i}\right), x\right)$ is a proof for $X$ and $F_{i}$ containing the same $x$.

Enroll $(p p, i k, p k)$ Parse $p k$ as $\left(X,\left(F_{i}, P_{i}\right)_{i=1}^{\Lambda}\right)$.

1. Check all proofs $P_{i}$; if one is invalid, return $\perp$.

2. $\operatorname{cert}_{i}:=\mathrm{FSig}\left(\omega, F_{i}\right)$ for $1 \leq i \leq \Lambda$.

3. Add $\left(X,\left(F_{i}, P_{i}, \text { cert }_{i}\right)_{i=1}^{\Lambda}\right)$ to UList and return $\left(\text { cert }_{i}\right)_{i=1}^{\Lambda}$.

The user defines her secret key as $s k:=\left(X,\left(F_{i}, P_{i}, \operatorname{cert}_{i}\right)_{i=1}^{\Lambda}, x\right)$.

$\operatorname{Dlg}\left(p p, s k_{i},\left[\right.\right.$ warr $\left.\left._{\rightarrow i}\right], p k_{i+1}\right) \quad$ Let the user holding $s k_{i}$ be the $i^{\text {th }}$ delegator.

1. Parse $s k_{i}$ as $\left(X_{i},\left(F_{i, j}, P_{i, j}, \operatorname{cert}_{i, j}\right)_{j=1}^{\Lambda}, x_{i}\right)$,

$p k_{i+1}$ as $\left(X_{i+1},\left(F_{i+1, j}, P_{i+1, j}\right)_{j=1}^{\Lambda}\right)$

and $\operatorname{warr}_{\rightarrow i}$ as $\left(\left(X_{j}, F_{j, j}, P_{j, j}, \operatorname{cert}_{j, j}, \sigma_{j}\right)_{j=1}^{i-1},\left(X_{i}^{\prime}, F_{i, i}^{\prime}, P_{i, i}^{\prime}\right)\right)$,

in case $i=1$, define warr $_{\rightarrow 1}:=\left(X_{1}, F_{1,1}, P_{1,1}\right)$

2. If one of the proofs in warr $\rightarrow i$ or $p k_{i+1}$ is invalid or if

$\left(X_{i}^{\prime}, F_{i, i}^{\prime}, P_{i, i}^{\prime}\right) \neq\left(X_{i}, F_{i, i}, P_{i, i}\right)$ then return $\perp$.

3. Define $\sigma_{i} \leftarrow \operatorname{FSig}\left(x_{i}, F_{1,1} \cdots F_{i, i} \cdot F_{i+1, i+1}\right)$.

Return warr $_{\rightarrow i+1}:=\operatorname{warr}_{\rightarrow i} \|\left(\operatorname{cert}_{i, i}, \sigma_{i},\left(X_{i+1}, F_{i+1, i+1}, P_{i+1, i+1}\right)\right)$.

$\operatorname{PSig}\left(p p, s k_{i}\right.$, warr $\left.\rightarrow i_{i}, M\right)$ Let the user holding $s k_{i}$ be the $(i-1)^{\text {st }}$ delegatee.

1. and 2. as for DIg (but ignoring the commands for $p k_{i+1}$ ).

3. Define $\sigma_{i}:=\operatorname{FSig}\left(x_{i}, F_{1,1} \cdots F_{i, i} \cdot \mathcal{F}_{\Lambda}(M)\right)$. The proxy signature is

$$
\Sigma:=\left(\sigma_{1},\left(X_{j}, F_{j, j}, P_{j, j}, \operatorname{cert}_{j, j}, \sigma_{j}\right)_{j=2}^{i}\right) .
$$

$\operatorname{PVer}(p p, p k, M, \Sigma)$ Let $p k=\left(X_{1}, F_{1,1}, P_{1,1}, ..\right), \Sigma=\left(\sigma_{1},\left(X_{i}, F_{i, i}, P_{i, i}, \operatorname{cert}_{i, i}, \sigma_{i}\right)_{i=2}^{k}\right)$. Return 0 if any of the following returns 0 , otherwise return 1 .

1. $\mathrm{V}_{\mathrm{X} \leftrightarrow \mathrm{F}}\left(\operatorname{crs}_{i},\left(X_{i}, F_{i, i}\right), P_{i, i}\right)$, for $1 \leq i \leq k$,

2. $\operatorname{FVer}\left(\Omega, F_{i, i}, \operatorname{cert}_{i, i}\right)$, for $2 \leq i \leq k$,

3. $\mathrm{FVer}\left(X_{i}, F_{1,1} \cdots F_{i+1, i+1}, \sigma_{i}\right)$, for $1 \leq i<k$,

$\operatorname{FVer}\left(X_{k}, F_{1,1} \cdots F_{k, k} \cdot \mathcal{F}_{\Lambda}(M), \sigma_{k}\right)$.

Open $(p p, p k, M, \Sigma$, UList $)$

If $\Sigma$ is valid, parse it as $\left(\sigma_{1},\left(X_{i}, F_{i, i}, P_{i, i}, \operatorname{cert}_{i, i}, \sigma_{i}\right)_{i=2}^{k}\right)$. If for all $i, X_{i} \in U L i s t$, return $\left(X_{2}, \ldots, X_{k}\right)$, otherwise return $\perp$.

\section{Claim 2. Scheme $\mathcal{D S}$ is non-frameable}

We give an overview of the proof and refer to Appendix D.2 for the quite technical proof. Our strategy is to reduce a "framing" proxy signature to a forgery of a Waters signature: An EUF-CMA adversary $\mathcal{B}$ against Waters' scheme receives a public key $X$ from its environment and sets out to simulate the nonframeability game for adversary $\mathcal{A}$ against $\mathcal{D S}$, setting $X$ as the public key of a random honest user $U^{*}$. Now to do so, without knowledge of the secret key, it must simulate the hash values $\left(F_{i}\right)$ corresponding to $X$. We define thus a sequence of indistinguishable games: The first game is the original non-frameability game. In the next one, we simulate the zK-proofs $\left(P_{i}\right)$ in the public key of $U^{*}$. In the third game, relying on the XF assumption, we substitute the $\left(F_{i}\right)$ by random values. Now the last game can be simulated by $\mathcal{B}$, given the fact that the signatures required to answer Dlg and PSig queries can be forwarded to $\mathcal{B}$ 's 
own signing oracle. If $\mathcal{A}$ wins the non-frameability game by framing $U^{*}$, then the signature output by $\mathcal{A}$ contains a Waters forgery.

However, to win the EUF-CMA game, $\mathcal{B}$ is required to return the bits of the message rather than its hash value - in fact, $\mathcal{B}$ 's oracle queries also require messages. This is why we need $\Pi_{\mathrm{X} \leftrightarrow \mathrm{F}}$ to be an extractable proof system; moreover, extraction must be possible even after having simulated proofs - which is the reason for $\Pi_{\mathrm{X} \leftrightarrow \mathrm{F}}$ to be weakly simulation sound.

\section{Claim 3. Scheme $\mathcal{D S}$ is traceable}

Proof. The claim follows by a reduction to unforgeability of the Waters signature scheme for messages of length $\Lambda \cdot m$ using the following fact:

Let $\mathbf{0}^{i}$ denote a string of $i \cdot m$ zeroes. Then for any $x \in\{0,1\}^{m}$ and any $i^{*}$, a signature on $\left(\mathbf{0}^{i^{*}-1}\|x\| \mathbf{0}^{\Lambda-i^{*}}\right)$ w.r.t. parameters $\left(\left(u_{i, j}\right)_{j=1}^{m}\right)_{i=1}^{\Lambda}$ is a signature on $x$ w.r.t. parameters $\left(u_{i^{*}, j}\right)_{j=1}^{\Lambda}$.

The simulator sets $\Omega$ to the public key it is challenged on and deals with Enroll $\left(X,\left(F_{i}, P_{i}\right)\right)$ queries as follows: If one of the $P_{i}$ is invalid, return $\perp$, otherwise extract $x$ from one of them. To produce cert $t_{i}$, query a signature on the message $\left(\mathbf{0}^{i-1}\|x\| \mathbf{0}^{\Lambda-i}\right)$. Open the signature returned by the adversary to $X_{2}, \ldots, X_{k}$. If $X_{i} \notin U L i s t$ for some $i$, return cert ${ }_{i}$ from the signature, together with the extracted bits.

\section{The Anonymous Delegation Scheme}

Now using the techniques derived from the Leak Tightness Lemma as discussed in Sect. 3, we can convert the scheme $\mathcal{D S}$ from the last section into an anonymous proxy signature scheme $\mathcal{A P S}$. We give the necessary modifications to $\mathcal{D} \mathcal{S}$ :

Setup $\left(1^{\lambda}, \Lambda\right)$ Choose a bilinear group of composite order $\left(p, q, \mathbb{G}, \mathbb{G}_{T}, e, g\right) \leftarrow \mathcal{G}_{c}\left(1^{\lambda}\right)$ and define $g$ Par $:=$ $\left(n=p q, \mathbb{G}, \mathbb{G}_{T}, e, g\right)$. Add $H \leftarrow \mathbb{G}_{q}$, a subgroup element for BGN-encryptions, to $p p$ and additionally output the opening key ok:=q.

Enroll $(p p, i k,(X, \ldots))$ The opener approves ${ }^{4}$ a new public key by verifying that $X^{q} \neq\left(X^{\prime}\right)^{q}$ for all $X^{\prime} \in U$ List before adding $X$ to UList.

$\operatorname{PSig}\left(p p, \operatorname{sk}_{k}\right.$, warr $\left._{\rightarrow k}, M\right)$ After producing $\Sigma=\left(\sigma_{1},\left(X_{i}, F_{i, i}, P_{i, i}, \text { cert }_{i, i}, \sigma_{i}\right)_{i=2}^{k}\right)$, blind $\Sigma$ by BGN-encrypting all elements of $\Sigma$ under $H$ and adding one proof $\pi$ (cf. Lemma 1) per pairing-product equation to be satisfied in PVer. Denote the result as $\widetilde{\Sigma}:=\left(\widetilde{\sigma}_{1},\left(\widetilde{X}_{i}, \widetilde{F}_{i, i}, \widetilde{P}_{i, i}, \widetilde{\operatorname{cert}}_{i, i}, \widetilde{\sigma}_{i}\right)_{i=2}^{k},\left(\pi_{i}\right)\right)$.

$\operatorname{PVer}(p p, p k, M, \widetilde{\Sigma})$ Instead of verifying the pairing-product equations directly, verify the proofs $\left(\pi_{i}\right)$ on the encrypted values.

Open(pp,ok, $M, \widetilde{\Sigma}$, UList $)$ If $\widetilde{\Sigma}$ passes verification, do the following for $2 \leq i \leq \Lambda$ : if $\widetilde{X}_{i}^{q}=\left(X^{\prime}\right)^{q}$ for some $X^{\prime} \in U$ List, then set $X_{i}:=X^{\prime}$, otherwise return $\perp$. Finally, return $\left(X_{2}, \ldots, X_{k}\right)$.

Anonymity. Consider two "plain" proxy signatures $\Sigma_{1}$ and $\Sigma_{2}$, both valid under the same public key and resulting from the same number of delegations (and consequently of the same size). If we blind both signatures and add proofs $\left(\pi_{i}\right)$, then they are indistinguishable: replacing $H$ by a random element in $\mathbb{G}$ is indistinguishable by SD. Now the signature components are perfectly blinded and the $\pi_{i}$ 's do not leak any information on the cleartexts besides validity by Lemma 1(2). As a consequence, $\mathcal{A P S}$ satisfies anonymity as defined in Sect. 3.3.

\footnotetext{
${ }^{4}$ If $X^{q}=\left(X^{\prime}\right)^{q}$ then the sets of ciphertexts of $X$ and $X^{\prime}$ coincide, making correct tracing impossible. Note that for random keys this is very improbable. It occurs if $X$ was maliciously set to $X^{\prime} H^{\rho}$ for some $\rho$, which makes the key useless anyway, as to compute the corresponding secret key one would have to know $\log _{g} H$.
} 
Traceability and Non-Frameability. Traceability and non-frameability both follow from a reduction to the respective notions for $\mathcal{D S}$ in the subgroup $\mathbb{G}_{p}$ using the techniques of Lemma 1. Given an adversary $\mathcal{A}$ against $\mathcal{A P S}$, we construct $\mathcal{B}$ against $\mathcal{D S}$ : After receiving $p p_{\mathcal{D S}}, \mathcal{B}$ defines $p p_{\mathcal{A P S}}$ by first creating parameters $p p^{\prime}, i k^{\prime}$ for a new instance of $\mathcal{D S}$ in group $\mathbb{G}_{q}$, and then multiplying all parameters from $p p_{\mathcal{D S}}$ with the new ones, resulting thus in correctly distributed parameters in $\mathbb{G}$, e.g., $g \in p p$ and $g^{\prime} \in p p^{\prime}$ yield $\bar{g}:=g g^{\prime} \in \mathbb{G}$. Finally, $\mathcal{B}$ adds $H \in \mathbb{G}_{q}$ to $p p_{\mathcal{A P S}}$. $\mathcal{A}$ 's oracle queries are dealt with in the following way:

PK queries. Run the PK oracle for $\mathcal{D S}$ to get $p k:=\left(X,\left(F_{i}, P_{i}\right)\right)$, then choose a secret key $x^{\prime} \in\{0,1\}^{m}$ and compute $X^{\prime}:=\left(g^{\prime}\right)^{x^{\prime}}$ and $F_{i}^{\prime}:=\prod\left(u_{i, j}^{\prime}\right)^{x_{j}^{\prime}}$ for $1 \leq i \leq \Lambda$, as well as the corresponding proofs w.r.t. parameters $p p^{\prime}$. Let the result be $p k^{\prime}$ and define $\left(\bar{X},\left(\bar{F}_{i}, \bar{P}_{i}\right)\right)$ by multiplying all components of $p k$ with the respective ones of $p k^{\prime}$.

First, note that due to Lemma 1(3), all proofs $\bar{P}_{i}$ satisfy all pairing-product equations of $\mathrm{V}_{\mathrm{X} \leftrightarrow \mathrm{F}}$. Second, $\left(\bar{X},\left(\bar{F}_{i}\right)\right)$ is indistinguishable from an honestly computed one by the XF-assumption in $\mathbb{G}, \mathbb{G}_{p}$ and $\mathbb{G}_{q} \cdot{ }^{5}$

Enroll, Dlg, PSig queries. Answering these queries basically consists of simulating $\operatorname{FSig}\left(y, F_{1} \cdots F_{k}\right)$ for some $y, F_{1}, \ldots, F_{k}$. Define $\theta_{p}, \theta_{q}$ such that $\theta_{p} \equiv_{p} 1, \theta_{p} \equiv_{q} 0, \theta_{q} \equiv_{p} 0, \theta_{q} \equiv_{q} 1$. If $F=\prod_{i=1}^{m}\left(u_{i} u_{i}^{\prime}\right)^{x_{i}}$, then $F^{\theta_{p}}=\prod u_{i}^{x_{i}} \in \mathbb{G}_{p}$ and $F^{\theta_{q}}=\prod\left(u_{i}^{\prime}\right)^{x_{i}} \in \mathbb{G}_{q}$. Now, $\mathcal{B}$ submits $F_{1}^{\theta_{p}} \cdots F_{k}{ }^{\theta_{p}}$ to its own oracle to get $\sigma$ and-knowing all secret keys for the $q$-components-computes $\sigma^{\prime}$ in $\mathbb{G}_{q}$ on its own. Finally, $\mathcal{B}$ returns $\bar{\sigma}=\sigma \cdot \sigma^{\prime}$ which is a valid signature according to Lemma $1(3)$.

When $\mathcal{A}$ eventually returns $(p k, M, \Sigma), \mathcal{B}$ "translates" the result back to $\mathbb{G}_{p}$ by raising everything to the power of $\theta_{p}$ and outputs it. It follows from Lemma $1(4)$ that $\mathcal{B}$ 's output passes verification. If $\mathcal{A}$ wins its game then so does $\mathcal{B}$ :

Traceability. If $\mathcal{A}$ wins the game then for some $i$ we have: $\forall X^{\prime} \in U_{\text {List }} \mathcal{A P S}_{\mathcal{S}}: \widetilde{X}_{i}^{q} \neq\left(X^{\prime}\right)^{q}$, which implies $\widetilde{X}_{i}^{\theta_{p}} \neq\left(X^{\prime}\right)^{\theta_{p}}$. On the other hand we have UList $_{\mathcal{D S}}=\left\{X^{\theta_{p}} \mid X \in\right.$ UList $\left._{\mathcal{A P S}}\right\}$. Together, this means $\tilde{X}_{i}^{\theta_{p}} \notin U$ List $_{\mathcal{D S}}$, the condition for $\mathcal{B}$ winning the game.

Non-frameability. Analogously: $\mathcal{A}$ wins the game if in the returned signature, there is one delegation step it has not queried. Since we compare "openings" of the signature and the warrants, the argument works as for traceability.

\section{The Proof of Equality of Exponent and Hash Preimage}

In order to construct $\Pi_{\mathrm{X} \leftrightarrow \mathrm{F}}$, as introduced in Sect. 4.2, we will use the following proof systems, detailed in Appendix A.

$\Pi_{1 \mathrm{~L}}$ A perfect WI (witness indistinguishable) proof system similar to the one from [GOS06a]: Given two triples, it proves that at least one of them is linear w.r.t. a given basis. We generalize their method, in that the bases for each triple are not necessarily the same.

$\Pi_{\mathbf{b} \text {,eq }}$ From $\Pi_{1 \mathrm{~L}}$ we directly derive a proof of the following: Given a Gos-commitment to some $x$ and a linear encryption of some $g^{y}$, prove that $x, y \in\{0,1\}$ and $x=y$.

$\Pi_{\mathbf{c X}}$ Given a vector of GOS-commitments to bits $\left(c_{i}\right)_{i=1}^{m}$ and $X \in \mathbb{G}, \Pi_{\mathrm{cX}}$ is a NIZK proof for the committed values being the bits of $\log X$.

\footnotetext{
${ }^{5}$ An element $\left(g^{x},\left(\mathcal{F}_{i}(x)\right)\right) \in \mathcal{L}_{\mathbb{G}}$ is indistinguishable from a random element in $\mathbb{G}^{\Lambda+1}$ by the XF-Assumption in $\mathbb{G}$. Now the latter is indistinguishable from elements $\left(g^{x} \cdot\left(g^{\prime}\right)^{x_{1}^{\prime}},\left(\mathcal{F}_{i}(x) \cdot\left(g^{\prime}\right)^{x_{2}^{\prime}}\right)\right.$ in $\mathcal{L}_{\mathbb{G}_{p}} \cdot \mathbb{G}_{q}^{\Lambda+1}$ by the XF-Assumption in $\mathbb{G}_{p}$, whereas the one in $\mathbb{G}_{q}$ guarantees indistinguishability of $\mathcal{L}_{\mathbb{G}_{p}} \cdot \mathbb{G}_{q}^{\Lambda+1}$ from $\mathcal{L}_{\mathbb{G}_{p}} \cdot \mathcal{L}_{\mathbb{G}_{q}} \cdot$
} 
$\Pi_{\mathbf{c F}}$ Given a vector of commitments to bits $\left(c_{i}\right)_{i=1}^{m}$ and $F \in \mathbb{G}, \Pi_{\mathrm{cF}}$ is a NIZK proof for the committed values being a hash preimage of $F$, i.e., if $c_{i}$ commits to $x_{i}$ for all $i$, then $F=\mathcal{F}\left(x_{1}, \ldots, x_{m}\right)$.

$\Pi_{\mathrm{G}}$ Given $\left(p k, p k^{\prime}, d, d^{\prime}, c k, c, v\right), \Pi_{\mathrm{G}}$ is a wi proof for either $d$ and $d^{\prime}$ being linear encryptions of the same message under $p k, p k^{\prime}$, resp., or $c$ being a commitment to $v$ under $c k$.

We will also use a one-time signature scheme $\mathcal{S}_{\text {ots }}=\left(\mathrm{KGen}_{\mathrm{ots}}, \mathrm{Sig}_{\mathrm{ots}}, \mathrm{Ver}_{\mathrm{ots}}\right)$ (cf. [Gro06] for an implementation). All verification procedures of the above systems consist exclusively of checking pairing-product equations. We give an overview of our construction and refer to Appendix B for the details.

Let $((X, F), x) \in R_{\mathrm{X} \leftrightarrow \mathrm{F}}$, i.e., $X=g^{x}$ and $F=\mathcal{F}(x)$. Aiming for an extractable proof, we first produce vectors of commitments $\boldsymbol{c}_{\boldsymbol{X}}$ and $\boldsymbol{c}_{\boldsymbol{F}}$ to the bits of $x$ and prove consistency with $X$ and $F$ via $\Pi_{\mathrm{cX}}$ and $\Pi_{\mathrm{cF}}$, resp. The proofs can be simulated by replacing the commitment keys for $c_{X}$ and $c_{F}$ by perfectly hiding keys. However, to achieve extraction-zero knowledge (EZK), we must extract from proofs queried to the oracle, even after replacing the CRS by a simulated one. We thus add linear encryptions $d_{i}^{\prime}$ and $d_{i}^{\prime \prime}$ under public keys $p k^{\prime}, p k^{\prime \prime}$ of the bits in $c_{X i}$ and $c_{F i}$, resp., and prove that we did so via $\Pi_{\mathrm{b}, \mathrm{eq}}$. At the same time this proves that $c_{X i}, c_{F i}$ are commitments to bits and that $d_{i}^{\prime}, d_{i}^{\prime \prime}$ are encryptions of either $g^{0}$ or $g^{1}$.

The latter enables us to ensure equality of the plaintexts in $d_{i}^{\prime}$ and $d_{i}^{\prime \prime}$ for all $i$ at once, by proving that $d_{P}^{\prime}:=\prod\left(d_{i}^{\prime}\right)^{2^{i-1}}$ and $d_{P}^{\prime \prime}:=\prod\left(d_{i}^{\prime \prime}\right)^{2^{i-1}}$ decrypt to the same plaintext. However, this proof must contain some kind of trapdoor, because in the proof of EzK, $d_{i}^{\prime}$ and $d_{i}^{\prime \prime}$ might contain different plaintexts. To do so, we borrow a trick Groth uses to build RCA-secure encryption in [Gro06]:

Add a commitment $c_{G}$ under key $c k_{G}$ of a signature verification key $v k_{G}$ to the CRS of $\Pi_{\mathrm{X} \leftrightarrow \mathrm{F}}$ and require the prover to choose a one-time signature key pair $(v k, s k)$, and to add $v k$ and a signature on $(X, F)$ to the proof. The proof of consistency of $d_{P}^{\prime}$ and $d_{P}^{\prime \prime}$ is a $\Pi_{\mathrm{G}}$ proof of $\left(p k^{\prime}, p k^{\prime \prime}, d_{P}^{\prime}, d_{P}^{\prime \prime}, c k_{G}, c_{G}, v k\right)$. Now we can (one-time) simulate proofs by choosing $v k:=v k_{G}$ and using the corresponding signing key which is unknown to the adversary.

\section{Acknowledgments}

This work was supported in part by EADS, the French ANR-07-SESU-008-01 PAMPA Project and the European Commission through Contract ICT-2007-216676 ECRYPT II.

\section{References}

[ASW00] N. Asokan, V. Shoup, and M. Waidner. Optimistic fair exchange of digital signatures. IEEE J. Selected Areas in Comm., 18(4), pp. 593-610, 2000.

[BCKL08] M. Belenkiy, M. Chase, M. Kohlweiss, A. Lysyanskaya. Non-interactive anonymous credentials. TCC '08 LCNS 4948, pp. 356-374. Springer Verlag, 2008.

[BMW03] M. Bellare, D. Micciancio, B. Warinschi. Foundations of group signatures: Formal definitions, simplified requirements, and a construction based on general assumptions. EUROCRYPT '03, LNCS 2656, pp. 614-629. Springer Verlag, 2003.

[BR93] M. Bellare, P. Rogaway. Random oracles are practical: a paradigm for designing efficient protocols. ACM Conference on Computer and Communications Security '93, pp. 62-73, ACM, 1993.

[BSZ05] M. Bellare, H. Shi, C. Zhang. Foundations of group signatures: The case of dynamic groups. In CT-RSA 2005, LNCS 3376, pp. 136-153. Springer Verlag, 2005.

[BFM88] M. Blum, P. Feldman, S. Micali. Non-interactive zero-knowledge and its applications. STOC '88, pp. 103-112, ACM, 1988.

[BPW03] A. Boldyreva, A. Palacio, B. Warinschi. Secure proxy signature schemes for delegation of signing rights. IACR ePrint Archive: Report 2003/096, 2003.

[BB04] D. Boneh, X. Boyen. Short signatures without random oracles. EUROCRYPT '04, LCNS 3027, pp. 56-73, Springer Verlag, 2004.

[BBS04] D. Boneh, X. Boyen, H. Shacham. Short group signatures. CRYPTO '04, LNCS 3152, pp. 43-55, Springer Verlag, 2004 
[BGLS03] D. Boneh, C. Gentry, B. Lynn, H. Shacham. Aggregate and verifiably encrypted signatures from bilinear maps. EUROCRYPT '03, LNCS 2656, pp. 416-432, Springer Verlag, 2003.

[BGN05] D. Boneh, E.-J. Goh, K. Nissim. Evaluating 2-DNF formulas on ciphertexts. TCC '05, LCNS 3378, pp. 325-341, Springer Verlag, 2005

[BW06] X. Boyen, B. Waters. Compact group signatures without random oracles. EUROCRYPT '06, LNCS 4004, pp. 427-444. Springer Verlag, 2006

[BW07] X. Boyen, B. Waters. Full-domain subgroup hiding and constant-size group signatures. PKC '07, LNCS 4450, pp. 1-15. Springer Verlag, 2007.

[Cha85] D. Chaum. Security without identication: transaction systems to make big brother obsolete. Communications of the ACM, 28(10), pp. 1030-1044, 1985.

[CvH91] D. Chaum, E. van Heyst. Group signatures. EUROCRYPT '91, LNCS 547, pp. 257-265. Springer Verlag, 1991.

[DP92] A. De Santis, G. Persiano. Zero-knowledge proofs of knowledge without interaction. FOCS '92, pp. 427-436, IEEE Computer Society, 1992.

[FP08a] G. Fuchsbauer, D. Pointcheval. Anonymous proxy signatures. SCN '08, LNCS 5229, pp. 201-217. Spinger Verlag, 2008

[GMR88] S. Goldwasser, S. Micali, R. Rivest. A digital signature scheme secure against adaptive chosen-message attacks. SIAM Journal on Computing, 17(2):281-308, 1988.

[GOS06a] J. Groth, R. Ostrovsky, A. Sahai. Non-interactive zaps and new techniques for NIZK. CRYPTO '06, LNCS 4117, pp. 97-111, Springer Verlag, 2006.

[GOS06b] J. Groth, R. Ostrovsky, A. Sahai. Perfect non-interactive zero knowledge for NP. EUROCRYPT '06, LNCS 4004, pp. 339-358, Springer Verlag, 2006.

[Gro07] J. Groth. Fully anonymous group signatures without random oracles. ASIACRYPT '07, LNCS 4833, pp. 164-180, Springer Verlag, 2007

[Gro06] J. Groth. Simulation-sound NIZK proofs for a practical language and constant size group signatures. ASIACRYPT '06, LCNS 4284, pp. 444-459, Springer Verlag, 2006

[GS08] J. Groth, A. Sahai. Efficient non-interactive proof systems for bilinear groups. EUROCRYPT '08, LCNS 4965, pp. 415-432, Springer Verlag, 2008.

[KP98] J. Kilian, E. Petrank. Identity escrow. CRYPTO '98: LCNS 1462, pp. 169-185, Springer Verlag, 1998.

[MUO96] M. Mambo, K. Usuda, E. Okamoto. Proxy signatures for delegating signing operation. Proceedings of the 3rd ACM Conference on Computer and Communications Security (CCS). ACM, 1996.

[Nao03] M. Naor. On cryptographic assumptions and challenges. CRYPTO '03, LCNS 2729, pp. 96-109, Springer Verlag, 2003.

[Sah99] A. Sahai. Non-malleable non-interactive zero knowledge and adaptive chosen-ciphertext security. FOCS '99, pp. 543-553, IEEE Computer Society, 1999.

[TW05] M. Trolin, D. Wikström. Hierarchical group signatures. Automata, Languages and Programming, 32nd International Colloquium (ICALP'05), LNCS 3580, pp. 446-458, Springer Verlag, 2005.

[Wat05] B. Waters. Efficient identity-based encryption without random oracles. EUROCRYPT '05 LNCS 3494, pp. 114127, Springer Verlag, 2005

\section{A Tools}

We give the proof systems introduced in Sect. 6 .

\section{A.1 $\Pi_{1 \mathrm{~L}}$, a Perfectly WI Proof of Linearity of One of Two Triples w.r.t. Different Bases}

[GOS06a] give a witness indistinguishable (WI) proof for one of two tuples being linear. We extend their ideas to construct a proof system where linearity holds w.r.t. different bases.

Let $\left(n, \mathbb{G}, \mathbb{G}_{T}, e\right)$ be a bilinear group, $f, h, g, \bar{f}, \bar{h}, \bar{g}$ be generators. Given two triples $c=\left(c_{1}, c_{2}, c_{3}\right)$ and $\bar{c}=\left(\bar{c}_{1}, \bar{c}_{2}, \bar{c}_{3}\right)$, we prove that either $c$ is linear w.r.t. $(f, h, g)$ or $\bar{c}$ is linear w.r.t. $(\bar{f}, \bar{h}, \bar{g})$.

Proof. In case $c=\left(f^{r}, h^{s}, g^{r+s}\right)$, let $\bar{r}, \bar{s}:=0$, in case $\bar{c}=\left(\bar{f}^{\bar{r}}, \bar{h}^{\bar{s}}, \bar{g}^{\bar{r}+\bar{s}}\right)$, let $r, s:=0$. Define

$$
\begin{array}{lllll}
\pi_{11}:=c_{1}^{\bar{r}} f^{t_{11}} & \pi_{12}:=c_{1}^{\bar{s}} f^{t_{12}} & \bar{\pi}_{11}:=\bar{c}_{1}^{r} \bar{f}^{-t_{11}} & \bar{\pi}_{12}:=\bar{c}_{2}^{r} \bar{h}^{-t_{12}} & \bar{\pi}_{13}:=\bar{c}_{3}^{r} \bar{g}^{-t_{11}-t_{12}} \\
\pi_{21}:=c_{2}^{\bar{r}} h^{t_{21}} & \pi_{22}:=c_{2}^{\bar{s}} h^{t_{22}} & \bar{\pi}_{21}:=\bar{c}_{1}^{s} \bar{f}^{-t_{21}} & \bar{\pi}_{22}:=\bar{c}_{2}^{s} \bar{h}^{-t_{22}} & \bar{\pi}_{23}:=\bar{c}_{3}^{s} \bar{g}^{-t_{21}-t_{22}} \\
\pi_{31}:=c_{3}^{\bar{r}} g^{t_{11}+t_{21}} & \pi_{32}:=c_{3}^{s} g^{t_{12}+t_{22}} & & &
\end{array}
$$


for random $t_{11}, t_{12}, t_{21}, t_{22}$.

Verification. Check the following relations:

$$
\begin{aligned}
& e\left(f, \bar{\pi}_{11}\right) e\left(\pi_{11}, \bar{f}\right)=e\left(c_{1}, \bar{c}_{1}\right) \quad e\left(f, \bar{\pi}_{12}\right) e\left(\pi_{12}, \bar{h}\right)=e\left(c_{1}, \bar{c}_{2}\right) \quad e\left(f, \bar{\pi}_{13}\right) e\left(\pi_{11} \pi_{12}, \bar{g}\right)=e\left(c_{1}, \bar{c}_{3}\right) \\
& e\left(h, \bar{\pi}_{21}\right) e\left(\pi_{21}, \bar{f}\right)=e\left(c_{2}, \bar{c}_{1}\right) \quad e\left(h, \bar{\pi}_{22}\right) e\left(\pi_{22}, \bar{h}\right)=e\left(c_{2}, \bar{c}_{2}\right) \quad e\left(h, \bar{\pi}_{23}\right) e\left(\pi_{21} \pi_{22}, \bar{g}\right)=e\left(c_{2}, \bar{c}_{3}\right) \\
& e\left(g, \bar{\pi}_{11} \bar{\pi}_{21}\right) e\left(\pi_{31}, \bar{f}\right)=e\left(c_{3}, \bar{c}_{1}\right) \quad e\left(g, \bar{\pi}_{12} \bar{\pi}_{22}\right) e\left(\pi_{32}, \bar{h}\right)=e\left(c_{3}, \bar{c}_{2}\right) \quad e\left(g, \bar{\pi}_{13} \bar{\pi}_{23}\right) e\left(\pi_{31} \pi_{32}, \bar{g}\right)=e\left(c_{3}, \bar{c}_{3}\right)
\end{aligned}
$$

Perfect Completeness. We content ourselves to show satisfaction of the relations "in the corner", for the rest works analogously. Note that the last equality in each line follows from the fact that either $r, s=0$ or $\bar{r}, \bar{s}=0$

$$
\begin{aligned}
& e\left(f, \bar{\pi}_{11}\right) e\left(\pi_{11}, \bar{f}\right)=e\left(f, \bar{c}_{1}^{r}\right) e\left(f, \bar{f}^{-t_{11}}\right) e\left(c_{1}^{\bar{r}}, \bar{f}\right) e\left(f^{t_{11}}, \bar{f}\right)=e\left(f^{r}, \bar{c}_{1}\right) e\left(c_{1}, \bar{f}^{\bar{r}}\right)=e\left(c_{1}, \bar{c}_{1}\right) \\
& e\left(f, \bar{\pi}_{13}\right) e\left(\pi_{11} \pi_{12}, \bar{g}\right)=e\left(f, \bar{c}_{3}^{r}\right) e\left(f, \bar{g}^{-t_{11}-t_{12}}\right) e\left(c_{1}^{\bar{r}+\bar{s}}, \bar{g}\right) e\left(f^{t_{11}+t_{12}}, \bar{g}\right)=e\left(f^{r}, \bar{c}_{3}\right) e\left(c_{1}, \bar{g}^{\bar{r}+\bar{s}}\right)=e\left(c_{1}, \bar{c}_{3}\right) \\
& e\left(g, \bar{\pi}_{11} \bar{\pi}_{21}\right) e\left(\pi_{31}, \bar{f}\right)=e\left(g, \bar{c}_{1}^{r+s}\right) e\left(g, \bar{f}^{-t_{11}-t_{21}}\right) e\left(c_{3}^{\bar{r}}, \bar{f}\right) e\left(g^{t_{11}+t_{21}}, \bar{f}\right)=e\left(g^{r+s}, \bar{c}_{1}\right) e\left(c_{3}, \bar{f}^{\bar{r}}\right)=e\left(c_{3}, \bar{c}_{1}\right) \\
& e\left(g, \bar{\pi}_{13} \bar{\pi}_{23}\right) e\left(\pi_{31} \pi_{32}, \bar{g}\right)=e\left(g, \bar{c}_{3}^{r+s}\right) e\left(g, \bar{g}^{-t_{11}-t_{12}-t_{21}-t_{22}}\right) e\left(c_{3}^{\bar{r}+\bar{s}}, \bar{g}\right) e\left(g^{t_{11}+t_{12}+t_{21}+t_{22}}, \bar{g}\right)=e\left(c_{3}, \bar{c}_{3}\right)
\end{aligned}
$$

Perfect Soundness. Define

$$
\begin{array}{llll}
\gamma_{1}:=\log _{f} c_{1} & \bar{\gamma}_{1}:=\log _{\bar{f}} \bar{c}_{1} & m_{1 j}:=\log _{f} \pi_{1 j} & \bar{m}_{i 1}:=\log _{\bar{f}} \bar{\pi}_{i 1} \\
\gamma_{2}:=\log _{h} c_{2} & \bar{\gamma}_{2}:=\log _{\bar{h}} \bar{c}_{2} & m_{2 j}:=\log _{h} \pi_{2 j} & \bar{m}_{i 2}:=\log _{\bar{h}} \bar{\pi}_{i 2} \\
\gamma_{3}:=\log _{g} c_{3} & \bar{\gamma}_{3}:=\log _{\bar{g}} \bar{c}_{3} & m_{3 j}:=\log _{g} \pi_{3 j} & \bar{m}_{i 3}:=\log _{\bar{g}} \bar{\pi}_{i 3}
\end{array}
$$

Then $e(f, \bar{f})^{m_{11}+\bar{m}_{11}}=e\left(f, \bar{\pi}_{11}\right) e\left(\pi_{11}, \bar{f}\right)=e\left(c_{1}, \bar{c}_{1}\right)=e(f, \bar{f})^{\gamma_{1} \bar{\gamma}_{1}}$, thus $m_{11}+\bar{m}_{11}=\gamma_{1} \bar{\gamma}_{1}$. For all verification relations we get thus:

$$
\begin{aligned}
& \bar{m}_{11}+m_{11}=\gamma_{1} \bar{\gamma}_{1} \quad \bar{m}_{12}+m_{12}=\gamma_{1} \bar{\gamma}_{2} \quad \bar{m}_{13}+m_{11}+m_{12}=\gamma_{1} \bar{\gamma}_{3} \\
& \bar{m}_{21}+m_{21}=\gamma_{2} \bar{\gamma}_{1} \quad \bar{m}_{22}+m_{22}=\gamma_{2} \bar{\gamma}_{2} \quad \bar{m}_{23}+m_{21}+m_{22}=\gamma_{2} \bar{\gamma}_{3} \\
& \bar{m}_{11}+\bar{m}_{21}+m_{31}=\gamma_{3} \bar{\gamma}_{1} \quad \bar{m}_{12}+\bar{m}_{22}+m_{32}=\gamma_{3} \bar{\gamma}_{2} \quad \bar{m}_{13}+\bar{m}_{23}+m_{31}+m_{32}=\gamma_{3} \bar{\gamma}_{3}
\end{aligned}
$$

Now, these relations imply that either $\gamma_{3}=\gamma_{1}+\gamma_{2}$ or $\bar{\gamma}_{3}=\bar{\gamma}_{1}+\bar{\gamma}_{2}$, since

$$
\begin{aligned}
\left(\gamma_{1}+\gamma_{2}-\gamma_{3}\right)\left(\bar{\gamma}_{1}+\bar{\gamma}_{2}-\bar{\gamma}_{3}\right) & =\gamma_{1} \bar{\gamma}_{1}+\gamma_{1} \bar{\gamma}_{2}-\gamma_{1} \bar{\gamma}_{3}+\gamma_{2} \bar{\gamma}_{1}+\gamma_{2} \bar{\gamma}_{2}-\gamma_{2} \bar{\gamma}_{3}-\gamma_{3} \bar{\gamma}_{1}-\gamma_{3} \bar{\gamma}_{2}+\gamma_{3} \bar{\gamma}_{3} \\
=\bar{m}_{11}+\bar{m}_{12}-\bar{m}_{13} & +\bar{m}_{21}+\bar{m}_{22}-\bar{m}_{23}-\left(\bar{m}_{11}+\bar{m}_{21}\right)-\left(\bar{m}_{12}+\bar{m}_{22}\right)+\bar{m}_{13}+\bar{m}_{23}=0
\end{aligned}
$$

Perfect Witness-Indistinguishability. Suppose, both $c$ and $\bar{c}$ are linear (otherwise, there is only one witness and we're done). Let $\pi_{i j}$ be proofs formed with witness $(r, s)$ (and $\left.\bar{r}=\bar{s}=0\right)$ and randomness $t_{i j}$, and let $\pi_{i j}^{\prime}$ be proofs formed via witness $(\bar{r}, \bar{s})$ and the following (equally distributed) randomness:

$$
\begin{array}{ll}
t_{11}^{\prime}:=t_{11}-r \bar{r} & t_{12}^{\prime}:=t_{12}-r \bar{s} \\
t_{21}^{\prime}:=t_{21}-s \bar{r} & t_{22}^{\prime}:=t_{22}-s \bar{s}
\end{array}
$$

Then we get (we dispense with the cases $\pi_{2 j}, \bar{\pi}_{2 j}$ as they work analogously to $\pi_{1 j}, \bar{\pi}_{1 j}$ )

$$
\begin{array}{lll}
\pi_{11}=f^{t_{11}}=f^{r \bar{r}+t_{11}^{\prime}}=\pi_{11}^{\prime} \quad \pi_{12}=f^{t_{12}}=f^{r \bar{s}+t_{12}^{\prime}}=\pi_{12}^{\prime} & \bar{\pi}_{11}=\bar{f}^{\bar{r} r-t_{11}}=\bar{f}^{-t_{11}^{\prime}}=\bar{\pi}_{11}^{\prime} \\
\bar{\pi}_{12}=\bar{f}^{\bar{s} r-t_{12}}=\bar{f}^{-t_{12}^{\prime}}=\bar{\pi}_{12}^{\prime} & \bar{\pi}_{13}=\bar{g}^{(\bar{r}+\bar{s}) r-t_{11}-t_{12}}=\bar{g}^{-t_{11}^{\prime}-t_{12}^{\prime}}=\bar{\pi}_{13}^{\prime} \\
\pi_{31}=g^{t_{11}+t_{21}}=g^{r \bar{r}+t_{11}^{\prime}+s \bar{r}+t_{21}^{\prime}}=\pi_{31}^{\prime} & \pi_{32}=g^{t_{12}+t_{22}}=g^{r \bar{s}+t_{12}^{\prime}+s \bar{s}+t_{22}^{\prime}=\pi_{32}^{\prime}}
\end{array}
$$




\section{A.2 $\Pi_{\mathrm{b}, \text { eq }}$, Proof for a Commitment and a Ciphertext Containing the Same Bit}

Using the WI proofs of the previous section, we can easily give a proof of the following: Given $c=$ $\operatorname{Com}(c k, x ; r, s)$ and $d=\operatorname{Enc}\left(p k, g^{x^{\prime}} ; r^{\prime}, s^{\prime}\right)$, with $c k=(v, w, u, f, h, z)$ and $p k=\left(f^{\prime}, h^{\prime}, z^{\prime}\right)$, prove that $x=x^{\prime}$ and $x \in\{0,1\}$.

$$
\begin{aligned}
& \mathrm{P}_{\mathrm{b}, \mathrm{eq}}\left(\operatorname{crs},(c, d),\left(x, r_{0}, s_{0}, r_{1}, s_{1}\right)\right):=\left(\pi_{1}, \pi_{2}\right) \text { with } \\
& \quad-\pi_{1}:=\mathrm{P}_{1 L}\left(\left(f, h, z, f^{\prime}, h^{\prime}, z^{\prime}\right),\left(\left(c_{1}, c_{2}, c_{3}\right),\left(d_{1}, d_{2}, d_{3} g^{-1}\right)\right),\left(r_{x}, s_{x}\right)\right) \\
& -\pi_{2}:=\mathrm{P}_{1 L}\left(\left(f, h, z, f^{\prime}, h^{\prime}, z^{\prime}\right),\left(\left(c_{1} v^{-1}, c_{2} w^{-1}, c_{3} u^{-1}\right),\left(d_{1}, d_{2}, d_{3}\right)\right),\left(r_{1-x}, s_{1-x}\right)\right)
\end{aligned}
$$

Now depending on whether $c k$ is binding or hiding, we get either soundness or simulation:

ck binding Suppose both proofs pass verification. (1) if $c$ is linear, $\left(c_{1} v^{-1}, c_{2} w^{-1}, c_{3} u^{-1}\right)$ is non-linear, so by $\pi_{2}, d$ must be linear; $c$ is thus a commitment to 0 , and $d$ an encryption of $g^{0}$. (2) if on the other hand $\left(d_{1}, d_{2}, d_{3} g^{-1}\right)$ is linear, $d$ is not, thus $\left(c_{1} v^{-1}, c_{2} w^{-1}, c_{3} u^{-1}\right)$ is linear, again by $\pi_{2}$; thus, $c$ is a commitment to 1 , and $d$ an encryption of $g^{1}$.

ck hiding Now if the commitment key is perfectly hiding and given $r_{v}, s_{w}$ such that $v=f^{r_{v}}, w=h^{s_{w}}$, the proof can be simulated given the randomness in $c$ only: let $c$ be a commitment to 0 using $(r, s)$, then $\operatorname{Sim}_{\mathrm{b}, \mathrm{eq}}\left(\left(c r s, r_{v}, s_{w}\right),(c, d),\left(r_{0}, s_{0}\right)\right):=\left(\pi_{1}, \pi_{2}\right)$ where $\pi_{1}$ is constructed using $(r, s)$ and $\pi_{2}$ using $\left(r-r_{v}, s-s_{w}\right)$.

\section{A.3 $\Pi_{\mathrm{cX}}$, Proof for Commitments to the Bits of a Logarithm}

Let $\left(n, \mathbb{G}, \mathbb{G}_{T}, e(\cdot, \cdot), g\right)$ be a bilinear group, let $(a, b, g)$ be a binding commitment key for base $(f, h, z)$. Let $X=g^{x},\left(c_{i}\right)_{i=0}^{m-1}$ be commitments to $x_{i} \in\{0,1\}$. We prove that $x=\sum x_{i} 2^{i}$.

CRS generation choose $\left(n, \mathbb{G}, \mathbb{G}_{T}, e(\cdot, \cdot), g\right) \leftarrow \mathcal{G}\left(1^{\lambda}\right) ; f, h \leftarrow \mathbb{G}^{*} ; r_{a}, s_{b} \leftarrow \mathbb{Z}_{p} ; a:=f^{r_{a}}, b=h^{s_{b}}$, $z:=g^{\left(r_{a}+s_{b}+1\right)^{-1}}$. Define $c k:=(f, h, z, a, b, g) ;$ return crs $:=\left(n, \mathbb{G}_{,} \mathbb{G}_{T}, e(\cdot, \cdot), c k\right)$.

Proof The witnesses are $\left(x_{i}, r_{i}, s_{i}\right)_{i=0}^{m-1}$ s.t. $c_{i}=\operatorname{Com}\left(c k, x_{i} ; r_{i}, s_{i}\right) ;$ let $x:=\sum x_{i} 2^{i}$. The proof is $(A, B, L)$ with $A:=a^{x}, B:=b^{x}, L:=z^{\sum r_{i} 2^{i}}$.

Verification given $\operatorname{crs}=\left(n, \mathbb{G}, \mathbb{G}_{t}, e(\cdot, \cdot), a, b, g, f, h, z\right)$, statement $\left(X,\left(c_{i}\right)\right)$ and proof $(A, B, L)$

- check $e(A, g) \stackrel{?}{=} e(a, X)$ and $e(B, g) \stackrel{?}{=} e(b, X)$

- $\operatorname{check} e\left(A^{-1} \prod c_{i, 1}^{2^{i}}, z\right) \stackrel{?}{=} e(f, L)$ and $e\left(B^{-1} \prod c_{i, 2}^{2^{i}}, z\right) \stackrel{?}{=} e\left(h, X^{-1} L^{-1} \prod c_{i, 3}^{2^{i}}\right)$

Completeness (V1): trivial.

$$
\begin{aligned}
& (\mathrm{V} 2): \quad e\left(A^{-1} \prod c_{i, 1}^{2^{i}}, z\right)=e\left(a^{-x} a^{\sum x_{i} 2^{i}} f^{\sum r_{i} 2^{i}}, z\right)=e\left(f^{\sum r_{i} 2^{i}}, z\right)=e(f, L) \\
& \begin{array}{r}
e\left(B^{-1} \prod c_{i, 2}^{2^{i}}, z\right)=e\left(b^{-x} b^{\sum x_{i} 2^{i}} h^{\sum s_{i} 2^{i}}, z\right) \\
=e\left(h, z^{\sum s_{i} 2^{i}}\right)= \\
=e\left(h, g^{-x} z^{-\sum r_{i} 2^{i}} g^{\sum x_{i} 2^{i}} z^{\sum\left(r_{i}+s_{i}\right) 2^{i}}\right)=e\left(h, X^{-1} L^{-1} \prod c_{i, 3}^{2^{i}}\right)
\end{array}
\end{aligned}
$$

Soundness Let $x=\log _{g} X$ and $(A, B, L)$ a proof that passes verification. From (V1) we have $A=a^{x}$ and $B=b^{x}$. Let $c_{i}=\operatorname{Com}\left(\alpha_{i} ; r_{i}, s_{i}\right)$; define $\alpha=\sum \alpha_{i} 2^{i}$ and consider (V3). From

$$
e(f, L)=e\left(A^{-1} \prod c_{i, 1}^{2^{i}}, z\right)=e\left(a^{-x} a^{\sum \alpha_{i} 2^{i}} f^{\sum r_{i} 2^{i}}, z\right)=e\left(f^{r_{a}(\alpha-x)+\sum r_{i} 2^{i}}, z\right)
$$

we get $L=z^{r_{a}(\alpha-x)+\sum r_{i} 2^{i}}$. Analogously we have

$$
e\left(B^{-1} \prod c_{i, 2}^{2^{i}}, z\right)=e\left(b^{-x} b^{\sum \alpha_{i} 2^{i}} h^{\sum s_{i} 2^{i}}, z\right)=e\left(h^{s_{b}(\alpha-x)+\sum s_{i} 2^{i}}, z\right)
$$


and on the other hand (let $g=z^{t}$ ),

$$
e\left(h, X^{-1} L^{-1} \prod c_{i, 3}^{2^{i}}\right)=e\left(h, g^{-x} z^{-r_{a}(\alpha-x)-\sum r_{i} 2^{i}} g^{\alpha} z^{\sum\left(r_{i}+s_{i}\right) 2^{i}}\right)=e\left(h, z^{t(\alpha-x)-r_{a}(\alpha-x)+\sum s_{i} 2^{i}}\right)
$$

Since by (V3), the leftmost terms in (1) and (2) are equivalent, considering the exponents, we get $\left(r_{a}+s_{b}-t\right)(\alpha-x)=0$, thus $\alpha=x$, otherwise $(a, b, g)$ were not a binding commitment key.

Zero Knowledge - simulated CRS: choose $\phi, \psi, r_{a}, s_{b} \leftarrow \mathbb{Z}_{p}$ and define $f:=g^{\phi}, h:=g^{\psi}, a:=f^{r_{a}}$, $b:=h^{s_{b}}, z:=g^{\left(r_{a}+s_{b}\right)^{-1}}$. The trapdoor is $\operatorname{tr}:=\left(\phi, \psi, r_{a}, s_{b}\right)$. Since $(a, b, g)$ in the simulated CRS is linear w.r.t $(f, h, z)$, while in the real one it is not, they are indistinguishable by the decisional linear assumption.

- simulated proof: given $X,\left(c_{i}\right)$; let $x:=\log _{g} X$ be unknown.

- define $A:=X^{\phi r_{a}}$ and $B:=X^{\psi s_{b}}$. (perfect simulations since $a=g^{\phi r_{a}}, b=g^{\psi s_{b}}$.)

- define $L:=\left(\prod c_{i, 1}^{2^{i} \phi^{-1}} X^{-r_{a}}\right)^{\left(r_{a}+s_{b}\right)^{-1}}$.

To see that The simulation is perfect, let $r_{i}, s_{i}$ s.t. $c_{i, 1}=f^{r_{i}}, c_{i, 2}=h^{s_{i}}$. Setting $\tilde{r}_{i}:=r_{i}-r_{a} x_{i}$ and $\tilde{s}_{i}:=s_{i}-s_{b} x_{i}$, we have $c_{i}=\left(f^{r_{a} x_{i}+\tilde{r}_{i}}, h^{s_{b} x_{i}+\tilde{s}_{i}}, z^{\left(r_{a}+s_{b}\right) x_{i}+\tilde{r}_{i}+\tilde{s}_{i}}\right)=\left(a^{x_{i}} f^{\tilde{r}_{i}}, b^{x_{i}} h^{\tilde{s}_{i}}, g^{x_{i}} z^{\tilde{r}_{i}+\tilde{s}_{i}}\right)$ and

$$
\begin{aligned}
L=\left(\prod c_{i, 1}^{2^{i} \phi^{-1}} X^{-r_{a}}\right)^{\left(r_{a}+s_{b}\right)^{-1}}=\left(f^{\sum r_{i} 2^{i}}\right)^{\phi^{-1}\left(r_{a}+s_{b}\right)^{-1}}\left(g^{\sum x_{i} 2^{i}}\right)^{-r_{a}\left(r_{a}+s_{b}\right)^{-1}} & = \\
z^{\sum r_{i} 2^{i}} z^{-r_{a} \sum x_{i} 2^{i}} & =z^{\sum\left(r_{i}-r_{a} x_{i}\right) 2^{i}}=z^{\sum \widetilde{r}_{i} 2^{i}}
\end{aligned}
$$

Remark 4. Proof system $\Pi_{\mathrm{cX}}$ can be used to construct a proof of knowledge of logarithm: given statement $X$ and witness $x=\sum_{i=0}^{m-1} x_{i} 2^{i}$ s.t. $X=g^{x}$, produce a crs for $\Pi_{\mathrm{cX}}$. Next, for all $0 \leq i<m$, define $c_{i}:=\operatorname{Com}\left(\operatorname{crs}, x_{i} ; r_{i}, s_{i}\right)$ and, via $\mathrm{P}_{1 \mathrm{~L}}\left(\ldots,\left(c_{i},\left(c_{i 1} a^{-1}, c_{i 2} b^{-1}, c_{i 3} g^{-1}\right)\right), \ldots\right)$, prove that the committed values are in $\{0,1\}$. Finally, run $\mathrm{P}_{\mathrm{cX}}\left(\operatorname{crs},\left(X,\left(c_{i}\right)\right), \ldots\right)$. Now from each $c_{i}$, we can extract the committed bit using extraction key $\left(\log _{e} f, \log _{e} h\right)$.

\section{A.4 $\Pi_{\mathrm{cF}}$, Proof for Commitments to the Bits of a Hash Preimage}

Let $\left(n, \mathbb{G}, \mathbb{G}_{T}, e, g\right)$ be a bilinear group, let $\left(u_{i}\right)_{i=1}^{m}$ be a basis for the Waters signature scheme, and let $\left(v_{i}, w_{i}, u_{i}, f_{i}, h_{i}, z_{i}\right)_{i=1}^{m}$ be a special binding commitment key as constructed below. Given a hash value $F$ and commitments $\left(c_{i}\right)_{i=1}^{m}$ to $x_{i} \in\{0,1\}$, we show that the $c_{i}$ are commitments to the bits of a preimage of $F$.

Common Reference String given $\left(n, \mathbb{G}, \mathbb{G}_{T}, e\right)$ and $\left(u_{i}\right)_{i=1}^{m} \in \mathbb{G}^{\lambda}$. Choose $\phi, \psi, r_{v}, s_{w} \leftarrow \mathbb{Z}_{p}$ and define for $1 \leq i \leq m: f_{i}:=u_{i}^{\phi}, h_{i}:=u_{i}^{\psi}, v_{i}:=f_{i}^{r_{v}}, w_{i}:=h_{i}^{s_{w}}, z_{i}:=u_{i}^{\left(r_{v}+s_{w}+1\right)^{-1}}$. The common reference string is crs $:=\left(n, \mathbb{G}, \mathbb{G}_{T}, e,\left(c k_{i}\right)_{i=1}^{m}\right)$ with $c k_{i}:=\left(f_{i}, h_{i}, z_{i}, v_{i}, w_{i}, u_{i}\right)$.

Proof let the witness be $x_{1}, \ldots, x_{m} \in\{0,1\}$, s.t. $F=\mathcal{F}\left(x_{1}, \ldots x_{m}\right)$ and $\left(r_{i}, s_{i}\right)$, the randomness used for $c_{i}$ (i.e., $\left.c_{i}=\operatorname{Com}\left(c_{i}, x_{i} ;\left(r_{i}, s_{i}\right)\right)\right)$. The proof is $\pi=(V, W, Z)$ with $V:=\prod v_{i}^{x_{i}}, W:=\prod w_{i}^{x_{i}}$ and $Z:=\prod z_{i}^{r_{i}}$.

Verification given crs, statement $\left(F,\left(c_{i}\right)\right)$ and proof $(V, W, Z)$, return 1 if the following hold

- $e\left(V, u_{1}\right) \stackrel{?}{=} e\left(F, v_{1}\right)$ and $e\left(W, u_{1}\right) \stackrel{?}{=} e\left(F, w_{1}\right)$

- $e\left(V^{-1} \prod c_{i, 1}, z_{1}\right) \stackrel{?}{=} e\left(f_{1}, Z\right)$ and $e\left(W^{-1} \prod c_{i, 2}, z_{1}\right) \stackrel{?}{=} e\left(h_{1}, F^{-1} Z^{-1} \prod c_{i, 3}\right)$

Simulation - simulated CRS: As the real CRS, except that $z_{i}:=u_{i}^{\left(r_{v}+s_{w}\right)^{-1}}$.

- simulated proof: given $F,\left(c_{i}\right)$, define $V:=F^{r_{v}}, W:=F^{r_{w}}$ and $Z:=\left(\prod c_{i, 1}^{\phi^{-1}} F^{-r_{v}}\right)^{\left(r_{v}+s_{w}\right)^{-1}}$

Note that $\Pi_{\mathrm{cX}}$ is a special case of $\Pi_{\mathrm{cF}}$, setting $u_{i}:=g^{2^{i-1}}$, since $\mathcal{F}(x)=\prod u_{i}^{x_{i}}=g^{\sum x_{i} 2^{i-1}}=X$. 


\section{A.5 $\Pi_{\mathrm{G}}$, Groth's WI Proof of Two Encryptions Having the Same Plaintext or a Commitment Containing a Specific Value}

Due to limited space we will use the following wi proof as a black box (cf. the full version of [Gro06] for an implementation that respects the pairing-product-equation paradigm): Given two encryptions $d, d^{\prime}$ under public keys for linear encryption $p k, p k^{\prime}$, resp., a commitment $c$ under commitment key $c k$, and $v$, a vector of group elements. Then $\Pi_{\mathrm{G}}$ enables to give a wI proof for "either $d$ and $d^{\prime}$ contain the same plaintext or $c$ is a commitment to $v "$.

The statement $\left(p k, p k^{\prime}, d, d^{\prime}, c k, c, v\right)$ can be proven using either witness $\left(m, r, r^{\prime}\right)$ s.t. $d=\operatorname{Enc}(p k, m ; r)$ and $d^{\prime}=\operatorname{Enc}\left(p k, m ; r^{\prime}\right)$ or witness $(v, r)$ s.t. $c=\operatorname{Com}(c k, v ; r)$.

\section{B The Proof System $\Pi_{\mathrm{X} \leftrightarrow \mathrm{F}}=\left(\mathrm{K}_{\mathrm{X} \leftrightarrow \mathrm{F}}, \mathrm{P}_{\mathrm{X} \leftrightarrow \mathrm{F}}, \mathrm{V}_{\mathrm{X} \leftrightarrow \mathrm{F}}, \operatorname{Sim}_{\mathrm{X} \leftrightarrow \mathrm{F}}, \operatorname{Ext}_{\mathrm{X} \leftrightarrow \mathrm{F}}\right)$}

\section{Reference String Generation $\mathrm{K}_{\mathrm{X} \leftrightarrow \mathrm{F}}\left(p, \mathbb{G}, \mathbb{G}_{T}, e, g, \boldsymbol{u}\right)$}

$$
\begin{aligned}
& \text { - choose } \phi, \psi, \xi_{1}^{\prime}, \xi_{2}^{\prime}, \xi_{1}^{\prime \prime}, \xi_{2}^{\prime \prime}, r_{a}, s_{b}, r_{v}, s_{w}, r_{G} \leftarrow \mathbb{Z}_{p} \\
& -\left(\operatorname{vk}_{G}, s k_{G}\right) \leftarrow \mathrm{KGen}_{\mathrm{ots}}\left(1^{\lambda}\right) ; c k_{G} \leftarrow \mathrm{KGen}_{\mathrm{Com}, \operatorname{binding}}\left(1^{\lambda}\right) ; c_{G} \leftarrow \operatorname{Com}\left(c k_{G}, v k_{G} ; r_{G}\right) \\
& -\operatorname{crs}_{G}^{\prime} \leftarrow \mathrm{K}_{G}\left(1^{\lambda}\right) \\
& \text { - define } \quad f:=g^{\phi} \quad h:=g^{\psi} \quad z:=g^{\left(r_{a}+s_{b}+1\right)^{-1}} \quad a:=f^{r_{a}} \quad b:=h^{s_{b}} \\
& f^{\prime}:=z^{\xi_{1}^{\prime}} \quad h^{\prime}:=z^{\xi_{2}^{\prime}} \quad f^{\prime \prime}:=z^{\xi_{1}^{\prime \prime}} \quad h^{\prime \prime}:=z^{\xi_{2}^{\prime \prime}} \\
& \text { for } 1 \leq i \leq m f_{i}:=u_{i}^{\phi} \quad h_{i}:=u_{i}^{\psi} \quad z_{i}:=u_{i}^{\left(r_{v}+s_{w}+1\right)^{-1}} v_{i}:=u_{i}^{r_{v}} \quad w_{i}:=u_{i}^{s_{w}} \\
& -c k_{X}:=(f, h, z, a, b, g), c k_{F i}:=\left(f_{i}, h_{i}, z_{i}, v_{i}, w_{i}, u_{i}\right), p k^{\prime}:=\left(f^{\prime}, h^{\prime}, z\right), p k^{\prime \prime}:=\left(f^{\prime \prime}, h^{\prime \prime}, z\right) . \\
& \operatorname{crs}_{G}:=\left(c k_{G}, c_{G}, \operatorname{crs}_{G}^{\prime}\right) \\
& \operatorname{crs}:=\left(c k_{X}, c k_{F}, p k^{\prime}, p k^{\prime \prime}, \operatorname{crs} G\right) \quad \operatorname{tr}:=\left(\phi, \psi, r_{a}, s_{b}, r_{v}, s_{w}, v k_{G}, s k_{G}, r_{G}\right) \quad e k:=\left(\xi_{1}^{\prime}, \xi_{2}^{\prime}, \xi_{1}^{\prime \prime}, \xi_{2}^{\prime \prime}\right)
\end{aligned}
$$

Proof $\mathrm{P}_{\mathrm{X} \leftrightarrow \mathrm{F}}(\operatorname{crs},(X, F), x) \quad$ - for all $1 \leq i \leq m$ do: choose $r_{X i}, s_{X i}, r_{F i}, s_{F i}, r_{i}^{\prime}, s_{i}^{\prime}, r_{i}^{\prime \prime}, s_{i}^{\prime \prime} \leftarrow \mathbb{Z}_{p}$

$$
\begin{aligned}
& c_{X i}:=\operatorname{Com}\left(c k_{X}, x_{i} ; r_{X i}, s_{X i}\right) \quad c_{F i}:=\operatorname{Com}\left(c k_{F i}, x_{i} ; r_{F i}, s_{F i}\right) \\
& d_{i}^{\prime}:=\operatorname{Enc}\left(p k^{\prime}, g^{x_{i}} ; r_{i}^{\prime}, s_{i}^{\prime}\right) \quad \pi_{i}^{\prime}:=\mathrm{P}_{\mathrm{b}, \mathrm{eq}}\left(\left(c k_{X}, p k^{\prime}\right),\left(c_{X i}, d_{i}^{\prime}\right),\left(r_{X i}, s_{X i}, r_{i}^{\prime}, s_{i}^{\prime}\right)\right) \\
& d_{i}^{\prime \prime}:=\operatorname{Enc}\left(p k^{\prime \prime}, g^{x_{i}} ; r_{i}^{\prime \prime}, s_{i}^{\prime \prime}\right) \quad \pi_{i}^{\prime \prime}:=\mathrm{P}_{\mathrm{b}, \text { eq }}\left(\left(c k_{F i}, p k^{\prime \prime}\right),\left(c_{F i}, d_{i}^{\prime \prime}\right),\left(r_{F i}, s_{F i}, r_{i}^{\prime \prime}, s_{i}^{\prime \prime}\right)\right) \\
& \text { - } \pi_{X}:=\mathrm{P}_{\mathrm{cX}}\left(c k_{X},\left(X, \boldsymbol{c}_{X}\right),\left(x, \boldsymbol{r}_{X}, \boldsymbol{s}_{X}\right)\right) \\
& \text { - } \pi_{F}:=\mathrm{P}_{\mathrm{cF}}\left(c_{F},\left(F, \boldsymbol{c}_{F}\right),\left(x, \boldsymbol{r}_{F}, \boldsymbol{s}_{F}\right)\right) \\
& \text { - }(v k, s k) \leftarrow \mathrm{K}_{\mathrm{ots}}\left(1^{\lambda}\right) ; \quad \sigma:=\operatorname{Sig}_{\mathrm{ots}}(\mathrm{sk},(X, F)) \\
& \text { - } d_{P}^{\prime}:=\prod\left(d_{i}^{\prime}\right)^{2^{i-1}} ; r_{P}^{\prime}:=\sum r_{i}^{\prime} 2^{i-1} ; s_{P}^{\prime}:=\sum s_{i}^{\prime} 2^{i-1} \text {; } \\
& d_{P}^{\prime \prime}:=\prod\left(d_{i}^{\prime \prime}\right)^{2^{i-1}} ; r_{P}^{\prime \prime}:=\sum r_{i}^{\prime \prime} 2^{i-1} ; s_{P}^{\prime \prime}:=\sum s_{i}^{\prime \prime} 2^{i-1} \\
& \text { - } \pi_{G}:=\mathrm{P}_{\mathrm{G}}\left(\operatorname{crs}_{G}^{\prime},\left(p k^{\prime}, p k^{\prime \prime}, d_{P}^{\prime}, d_{P}^{\prime \prime}, c k_{G}, c_{G}, v k\right),\left(X, r_{P}^{\prime}, s_{P}^{\prime}, r_{P}^{\prime \prime}, s_{P}^{\prime \prime}\right)\right)\left(^{6}\right)
\end{aligned}
$$

The proof for $(X, F)$ is $\pi:=\left(\pi_{X}, \boldsymbol{c}_{X}, \boldsymbol{\pi}^{\prime}, \boldsymbol{d}^{\prime}, \pi_{G}, \boldsymbol{d}^{\prime \prime}, \boldsymbol{\pi}^{\prime \prime}, \boldsymbol{c}_{F}, \pi_{F}, v k, \sigma\right)$

Verification $\mathrm{V}_{\mathrm{X} \leftrightarrow \mathrm{F}}(\operatorname{crs},(X, F), \pi)$ To verify a proof, verify $\sigma$ on $(X, F)$ under vk and verify the proofs $\pi_{X}, \pi_{F}, \pi_{G}$ and $\pi_{i}^{\prime}, \pi_{i}^{\prime \prime}$ for all $1 \leq i \leq m$.

Extraction Ext $\operatorname{Ex}_{\leftrightarrow \mathrm{F}}(e k,(X, F), \pi)$ Let $e k:=\left(\xi^{\prime}, \xi^{\prime \prime}\right), \pi:=\left(\pi_{X}, \boldsymbol{c}_{X}, \boldsymbol{\pi}^{\prime}, \boldsymbol{d}^{\prime}, \pi_{G}, \boldsymbol{d}^{\prime \prime}, \boldsymbol{\pi}^{\prime \prime}, \boldsymbol{c}_{F}, \pi_{F}, v k, \sigma\right)$ If $\pi$ is valid, extract bits $x_{i}$ using either $\xi^{\prime}$ on $d_{i}^{\prime}$ or $\xi^{\prime \prime}$ on $d_{i}^{\prime \prime}$ for all $i$.

\footnotetext{
${ }^{6}$ Due to the homomorphic property of linear encryption, $d_{i}^{\prime}=\operatorname{Enc}\left(x_{i} ; r_{i}^{\prime}, s_{i}^{\prime}\right)$ implies $d_{P}^{\prime}=\operatorname{Enc}\left(\prod g^{x_{i} 2^{i-1}} ; r_{P}^{\prime}, s_{P}^{\prime}\right)$.
} 
Simulation $\operatorname{Sim}_{\mathrm{X} \leftrightarrow \mathrm{F}, 1}$ works as $\mathrm{K}_{\mathrm{X} \leftrightarrow \mathrm{F}}$ except for outputting $t r$ and replacing $c k_{X}$ and all $c k_{F i}$ by perfectly hiding commitment keys by setting $z:=g^{\left(r_{a}+s_{b}\right)^{-1}}$ and $z_{i}:=u_{i}^{\left(r_{v}+s_{w}\right)^{-1}}$

$\operatorname{Sim}_{\mathrm{X} \leftrightarrow \mathrm{F}, 2}-$ replace $c_{X i}$ and $c_{F i}$ by commitments to $0, d_{i}^{\prime}, d_{i}^{\prime \prime}$ by encryptions of $g^{0}$.

$$
\begin{aligned}
& -\pi_{X}:=\operatorname{Sim}_{\mathrm{cX}}\left(\left(\phi, \psi, r_{a}, r_{b}\right),\left(X, c_{X}\right)\right), \quad \pi_{i}^{\prime}:=\operatorname{Sim}_{\mathrm{b}, \mathrm{eq}}\left(\left(c k_{X}, p k^{\prime}, r_{a}, s_{b}\right),\left(c_{X i}, d_{i}^{\prime}\right),\left(r_{X i}, s_{X i}\right)\right) \\
& -\pi_{F}:=\operatorname{Sim}_{c F}\left(\left(\phi, \psi, r_{v}, s_{w}\right),\left(F, \boldsymbol{c}_{F}\right)\right), \quad \pi_{i}^{\prime \prime}:=\operatorname{Sim}_{\mathrm{b}, \mathrm{eq}}\left(\left(c k_{F i}, p k^{\prime \prime}, r_{v}, s_{w}\right),\left(c_{F i}, d_{i}^{\prime \prime}\right),\left(r_{F i}, s_{F i}\right)\right) \\
& -(v k, s k):=\left(v k_{G}, s k_{G}\right), \quad \sigma:=\operatorname{Sig}_{\mathrm{ots}}\left(s k_{G},(X, F)\right), \\
& \quad \pi_{G}:=\mathrm{P}_{G}\left(c \operatorname{crs}_{G},\left(p k^{\prime}, p k^{\prime \prime}, \prod\left(d_{i}^{\prime}\right)^{2^{i-1}}, \prod\left(d_{i}^{\prime \prime}\right)^{2^{i-1}}, c k_{G}, c_{G}, v k_{G}\right),\left(v k_{G}, r_{G}\right)\right)
\end{aligned}
$$

Soundness / Extraction. Let $\pi$ be a valid proof for $(X, F)$. First of all, soundness of $\pi_{i}^{\prime}$ guarantees that if $c_{X i}$ is a commitment to $x_{i}^{\prime}$, then $x_{i}^{\prime} \in\{0,1\}$ and furthermore $d_{i}^{\prime}$ encrypts $g^{x_{i}^{\prime}}$. Now $\pi_{X}$ ensures that $X=g^{\sum x_{i}^{\prime} i^{i-1}}$. An analogous argument applied to $\pi^{\prime \prime}$ and $\pi_{F}$ yields that if $c_{F i}$ commits to $x_{i}^{\prime \prime}$, then $d_{i}^{\prime \prime}$ encrypts $g^{x_{i}^{\prime \prime}}$, with $x_{i}^{\prime \prime} \in\{0,1\}$, and $F=\mathcal{F}\left(x_{1}^{\prime \prime}, \ldots, x_{m}^{\prime \prime}\right)$. Since the commitment $c_{G}$ is computationally hiding, it is only with negligible probability that vk is the committed value. Soundness of $\pi_{G}$ ensures thus that the values encrypted in $\prod\left(d_{i}^{\prime}\right)^{2^{i-1}}$ and $\prod\left(d_{i}^{\prime \prime}\right)^{2^{i-1}}$ are the same, i.e., $g^{\sum x_{i}^{\prime} 2^{i-1}}=g^{\sum x_{i}^{\prime \prime} 2^{i-1}}$, which, given the fact that $x_{i}^{\prime}, x_{i}^{\prime \prime} \in\{0,1\}$, means $x_{i}^{\prime}=x_{i}^{\prime \prime}$ for all $i$. Thus, $(X, F) \in \mathcal{L}_{\mathrm{X} \leftrightarrow \mathrm{F}}$ and the bits extracted by Ext are the bits of the witness.

\section{Extraction Zero Knowledge.}

Theorem $1 \Pi_{X \leftrightarrow F}$ is an extraction-zero-knowledge proof of knowledge and language membership.

Proof (sketch). We define a sequence of games:

GAme $0:=\operatorname{Exp}_{\Pi, \mathcal{A}}^{\mathrm{zk}}$, where Ext queries are answered using $\xi^{\prime}$. In addition, whenever $\mathcal{A}_{2}$ makes a valid query involving $(X, F)$, the statement output by $\mathcal{A}_{1}$ together with a witness $x$, we simply output $x$. Since $x$ is the only witness, Game 0 and $\operatorname{Exp}_{\Pi, \mathcal{A}}^{\mathrm{zk}}$ are indistinguishable by correctness of extraction.

GAME 1 Define $c r s$ to be a simulated reference string (triples $(a, b, g)$ and $\left(v_{i}, w_{i}, u_{i}\right)$ become linear w.r.t. $(f, h, e)$ and $\left(f_{i}, h_{i}, e_{i}\right)$, respectively. Indistinguishability of Games 0 and 1 is implied by the decisional linear assumption.

GAme 2 In $\mathrm{P}_{\mathrm{X} \leftrightarrow \mathrm{F}}(\operatorname{crs},(X, F))$, set $(v k, s k):=\left(v k_{G}, s k_{G}\right)$, the pair from tr, instead of running $\mathrm{K}_{\text {ots. }}$. Games 1 and 2 are indistinguishable by the computational hiding property of $c_{G}$.

Game 3 Compute $\pi$ by $\operatorname{Sim}_{\mathrm{X} \leftrightarrow \mathrm{F}, 2}$, except that $d_{i}^{\prime}, d_{i}^{\prime \prime}$ remain encryptions of $x_{i}$. The probabilities of Game 2 and 3 are equivalent, since all we did is compute the wI proofs using different witnesses: Commitments $c_{X i}$ and $c_{F i}$ are already linear in Game 2 due to the simulated CRs. Proofs $\pi_{X}, \pi_{F}, \pi_{i}^{\prime}$ and $\pi_{i}^{\prime \prime}$ are computed using different witnesses.

GAME 4 We replace encryptions $d_{i}^{\prime \prime}$ by encryptions of 0 . Games 3 and 4 are indistinguishable by a hybrid argument on semantic security of encryptions. (Note that the distinguisher can perfectly simulate both games, since we use $\xi^{\prime}$ to extract and because the WI proofs do not use the witnesses of $d_{i}^{\prime \prime}$.)

Game 5 The Ext-oracle uses $\xi^{\prime \prime}$ instead of $\xi^{\prime}$. The only difference between Games 4 and 5 would be if the adversary queried some $\left(\left(X^{*}, F^{*}\right), \pi^{*}\right)$ with $\left(X^{*}, F^{*}\right) \neq(X, F)$ and $\pi^{*}$ containing $\left(d_{i}^{\prime}\right)^{*}$ encrypting $x_{i}^{\prime}$ and $\left(d_{i}^{\prime \prime}\right)^{*}$ encrypting $x_{i}^{\prime \prime} \neq x_{i}^{\prime}$ for some $i$. However, in this case $\prod\left(\left(d_{i}^{\prime}\right)^{*}\right)^{2^{i-1}}$ and $\prod\left(\left(d_{i}^{\prime \prime}\right)^{*}\right)^{2^{i-1}}$ contain different ciphertexts. Now soundness of $\pi_{G}^{*}$ implies that $v k^{*}=v k_{G}$, while on the other hand $\sigma^{*}$ is a signature on $\left(X^{*}, F^{*}\right) \neq(X, F)$ (the statement output by $\mathcal{A}_{1}$ ), which is valid w.r.t. $v k_{G}$. The distance between Games 4 and 5 is thus upper-bounded by the advantage of a forger against the one-time signature scheme

Game 6 Replace encryptions $d_{i}^{\prime}$ by encryptions to 0 . Game 5 and 6 are indistinguishable analogously to Game 3 and 4. Game 6 is indistinguishable from $\operatorname{Exp}_{\Pi, \mathcal{A}}^{\mathrm{zk}-\mathrm{S}}$. 


\section{Security Definitions for Anonymous Proxy Signatures}

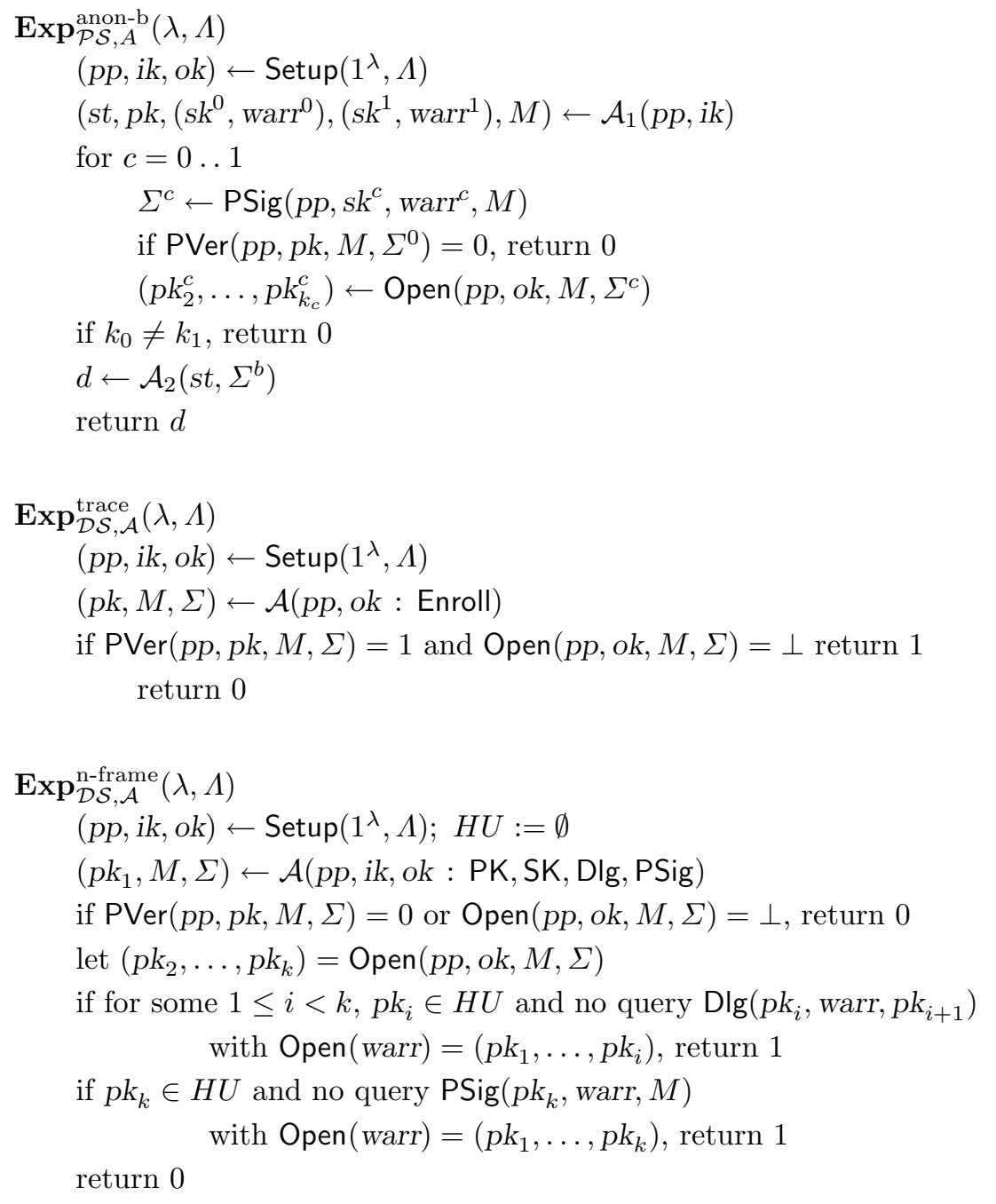

Figure 1. Experiments for anonymity, traceability and non-frameability

We review the slightly adapted security notions from [FP08a]. In particular, we do not consider delegation for specific tasks (although this can be easily included in our scheme, cf. Remark 3), we content ourselves to having only one general opener and our version of anonymity is CPA rather than CCA-2 as in their model.

Traceability and non-frameability are defined as any adversary being able to win games $\mathbf{E x p}^{\text {trace }}$, $\mathbf{E x p}^{\text {n-frame }}$, resp., with at most negligible probability. Anonymity holds if no adversary can distinguish $\mathbf{E x p}^{\text {anon-0 }}$ from $\mathbf{E x p}^{\text {anon-1 }}$ (see Fig. 1 for the definitions of the experiments).

In the experiments, the adversary disposes over a subset of the following oracles:

Enroll(pk) Enroll pk using the issuer's key.

PK Create a random public/private key pair $(p k, s k)$ and add it to $H U$, the list of honest users; output $p k$. 
$\mathrm{SK}(p k)$ If $p k$ is in $H U$, delete the entry and return the corresponding sk.

$\operatorname{Dlg}\left(p k, w a r r, p k^{\prime}\right)$ If $p k$ is in $H U$, look up the corresponding sk and return $\operatorname{Dlg}\left(p p, s k, w a r r, p k^{\prime}\right)$. Otherwise return $\perp$.

PSig $(p k$, warr, $M)$ If $p k$ is in $H U$, look up the corresponding sk and return PSig $(p p, s k$, warr, $M)$. Otherwise return $\perp$.

Definition 5 (Anonymity). A signature delegation scheme $\mathcal{D S}$ is anonymous if for any p.p.t. adversary $\mathcal{A}=\left(\mathcal{A}_{1}, \mathcal{A}_{2}\right)$, the following is negligible in $\lambda$ :

$$
\left|\operatorname{Pr}\left[\operatorname{Exp}_{\mathcal{P S}, \mathcal{A}}^{\text {anon-1 }}(\lambda)=1\right]-\operatorname{Pr}\left[\operatorname{Exp}_{\mathcal{P S}, \mathcal{A}}^{\text {anon- }}(\lambda)=1\right]\right|
$$

Definition 6 (Traceability). A signature delegation scheme $\mathcal{D S}$ is traceable if for any p.p.t. adversary $\mathcal{A}, \operatorname{Pr}\left[\operatorname{Exp}_{\mathcal{P} \mathcal{S}, \mathcal{A}}^{\text {trace }}(\lambda)=1\right]$ is negligible in $\lambda$.

Definition 7 (Non-frameability). A signature delegation scheme $\mathcal{D S}$ is non-frameable if for any p.p.t. adversary $\mathcal{A}, \operatorname{Pr}\left[\operatorname{Exp}_{\mathcal{P} \mathcal{S}, \mathcal{A}}^{\text {n-frame }}(\lambda)=1\right]$ is negligible in $\lambda$.

\section{Proofs}

\section{D.1 Proof of Lemma 1}

Proof of Lemma 1(1). Follows from bilinearity of $e(\cdot, \cdot)$.

Proof of Lemma 1(3). Consider $E_{\left(a_{j} c_{j}, b_{j} d_{j}\right)_{j}}$ :

$$
\begin{aligned}
& \prod e\left(a_{j} c_{j} \prod\left(X_{i} Y_{i}\right)^{\delta_{j, i}}, b_{j} d_{j} \prod\left(X_{i} Y_{i}\right)^{\varepsilon_{j, i}}\right)= \\
& \prod e\left(a_{j} \prod X_{i}^{\delta_{j, i}}, b_{j} \prod X_{i}^{\varepsilon_{j, i}}\right) e\left(a_{j} \prod X_{i}^{\delta_{j, i}}, d_{j} \prod Y_{i}^{\varepsilon_{j, i}}\right) e\left(c_{j} \prod Y_{i}^{\delta_{j, i}}, b_{j} \prod X_{i}^{\varepsilon_{j, i}}\right) e\left(c_{j} \prod Y_{i}^{\delta_{j, i}}, d_{j} \prod Y_{i}^{\varepsilon_{j, i}}\right)= \\
& \prod e\left(a_{j} \prod X_{i}^{\delta_{j, i}}, b_{j} \prod X_{i}^{\varepsilon_{j, i}}\right) \prod e\left(c_{j} \prod Y_{i}^{\delta_{j, i}}, d_{j} \prod Y_{i}^{\varepsilon_{j, i}}\right)=1
\end{aligned}
$$

where the second equation holds, because the two pairings in the middle of the second line are pairings of elements of $\mathbb{G}_{p}$ with elements of $\mathbb{G}_{q}$ and are therefore 1 .

Proof of Lemma 1(4). Let $\left(\widetilde{X}_{i}\right)$ satisfy $\tilde{E}_{\left(a_{j} c_{j}, b_{j} d_{j}\right)_{j}} . H$ is of order $q$ and consequently so is the right hand side of the equation, thus raising $(\widetilde{E})$ to the power of $\theta^{2}$ yields

$$
\mathbf{1}=e\left(H^{\theta}, P_{E}^{\theta}\right)=\prod_{j} e\left(\left(a_{j} c_{j}\right)^{\theta} \prod_{i} \widetilde{X}_{i}^{\theta \delta_{j, i}},\left(b_{j} d_{j}\right)^{\theta} \prod_{j} \widetilde{X}_{i}^{\theta \varepsilon_{j, i}}\right)=\prod_{j} e\left(a_{j} \prod_{i}\left(\widetilde{X}_{i}^{\theta}\right)^{\delta_{j, i}}, b_{j} \prod_{j}\left(\widetilde{X}_{i}^{\theta}\right)^{\varepsilon_{j, i}}\right)
$$

Proof of Lemma 1(2). Let $g$ be a generator of $\mathbb{G}$. For all $i$, define: $x_{i}:=\log _{g} X_{i}, x_{i}^{\prime}:=\log _{g} X_{i}^{\prime}, \alpha_{i}:=\log _{g} a_{i}$, $\beta_{i}:=\log _{g} b_{i}$ and $\kappa:=\log _{g} H$. Note that for all $i, X_{i} H^{\rho_{i}}=X_{i}^{\prime} H^{\rho_{i}^{\prime}}$ implies

$$
x_{i}^{\prime}-x_{i}=\kappa\left(\rho_{i}-\rho_{i}^{\prime}\right) .
$$

We show that $\Delta:=\log _{g} P_{E}\left(\left(X_{i}\right),\left(\rho_{i}\right)\right)-\log _{g} P_{E}\left(\left(X_{i}^{\prime}\right),\left(\rho_{i}^{\prime}\right)\right)=0$.

$$
\begin{aligned}
& \Delta=\sum_{j}\left(\left(\alpha_{j}+\sum_{i} \delta_{j, i} x_{i}\right)\left(\sum_{i} \varepsilon_{j, i} \rho_{i}\right)+\left(\beta_{j}+\sum_{i} \varepsilon_{j, i} x_{i}\right)\left(\sum_{i} \delta_{j, i} \rho_{i}\right)+\kappa\left(\sum_{i} \delta_{j, i} \rho_{i}\right)\left(\sum_{i} \varepsilon_{j, i} \rho_{i}\right)\right. \\
&\left.-\left(\alpha_{j}+\sum_{i} \delta_{j, i} x_{i}^{\prime}\right)\left(\sum_{i} \varepsilon_{j, i} \rho_{i}^{\prime}\right)-\left(\beta_{j}+\sum_{i} \varepsilon_{j, i} x_{i}^{\prime}\right)\left(\sum_{i} \delta_{j, i} \rho_{i}^{\prime}\right)-\kappa\left(\sum_{i} \delta_{j, i} \rho_{i}^{\prime}\right)\left(\sum_{i} \varepsilon_{j, i} \rho_{i}^{\prime}\right)\right)=A+B
\end{aligned}
$$


with $A:=\sum\left(\alpha_{j} \sum_{i} \varepsilon_{j, i}\left(\rho_{i}-\rho_{i}^{\prime}\right)+\beta_{j} \sum_{i} \delta_{j, i}\left(\rho_{i}-\rho_{i}^{\prime}\right)\right)$ and

$B:=\sum\left(\sum_{i} \delta_{j, i}\left(x_{i}+\kappa \rho_{i}\right) \sum_{i} \varepsilon_{j, i} \rho_{i}-\sum_{i} \delta_{j, i}\left(x_{i}^{\prime}+\kappa \rho_{i}^{\prime}\right) \sum_{i} \varepsilon_{j, i} \rho_{i}^{\prime}+\sum_{i} \varepsilon_{j, i} x_{i} \sum_{i} \delta_{j, i} \rho_{i}-\sum_{i} \varepsilon_{j, i} x_{i}^{\prime} \sum_{i} \delta_{j, i} \rho_{i}^{\prime}\right)$.

Considering the logarithms of $E\left(\left(X_{i}\right)\right)$ and $E\left(\left(X_{i}^{\prime}\right)\right)$ we get

$$
\sum\left(\alpha_{j}+\sum_{i} \delta_{j, i} x_{i}\right)\left(\beta_{j}+\sum_{i} \varepsilon_{j, i} x_{i}\right)=0=\sum\left(\alpha_{j}+\sum_{i} \delta_{j, i} x_{i}^{\prime}\right)\left(\beta_{j}+\sum_{i} \varepsilon_{j, i} x_{i}^{\prime}\right)
$$

which, subtracting the left-hand from the right-hand side, yields

$$
\sum\left(\alpha_{j} \sum_{i} \varepsilon_{j, i}\left(x_{i}^{\prime}-x_{i}\right)+\beta_{j} \sum_{i} \delta_{j, i}\left(x_{i}^{\prime}-x_{i}\right)+\sum_{i} \delta_{j, i} x_{i}^{\prime} \sum_{i} \varepsilon_{j, i} x_{i}^{\prime}-\sum_{i} \delta_{j, i} x_{i} \sum_{i} \varepsilon_{j, i} x_{i}\right)=0
$$

and from (3): $\sum\left(\kappa\left(\alpha_{j} \sum_{i} \varepsilon_{j, i}\left(\rho_{i}-\rho_{i}^{\prime}\right)+\beta_{j} \sum_{i} \delta_{j, i}\left(\rho_{i}-\rho_{i}^{\prime}\right)\right)+\sum_{i} \delta_{j, i} x_{i}^{\prime} \sum_{i} \varepsilon_{j, i} x_{i}^{\prime}-\sum_{i} \delta_{j, i} x_{i} \sum_{i} \varepsilon_{j, i} x_{i}\right)=0$

thus $\quad A=\frac{1}{\kappa} \sum\left(\sum_{i} \delta_{j, i} x_{i} \sum_{i} \varepsilon_{j, i} x_{i}-\sum_{i} \delta_{j, i} x_{i}^{\prime} \sum_{i} \varepsilon_{j, i} x_{i}^{\prime}\right)$.

On the other hand, since $x_{i}+\kappa \rho_{i}=x_{i}^{\prime}+\kappa \rho_{i}^{\prime}$, (note that we subtract and add $\sum \delta_{j, i} \rho_{i} \sum \varepsilon_{j, i} x_{i}^{\prime}$ )

$$
\begin{aligned}
B & =\sum\left(\sum_{i} \delta_{j, i}\left(x_{i}+\kappa \rho_{i}\right) \sum_{i} \varepsilon_{j, i}\left(\rho_{i}-\rho_{i}^{\prime}\right)-\sum_{i} \delta_{j, i} \rho_{i} \sum_{i} \varepsilon_{j, i}\left(x_{i}^{\prime}-x_{i}\right)+\sum_{i} \varepsilon_{j, i} x_{i}^{\prime} \sum_{i} \delta_{j, i}\left(\rho_{i}-\rho_{i}^{\prime}\right)\right) \\
& =\sum\left(\frac{1}{\kappa} \sum_{i} \delta_{j, i}\left(x_{i}+\kappa \rho_{i}\right) \sum_{i} \varepsilon_{j, i}\left(x_{i}^{\prime}-x_{i}\right)-\sum_{i} \delta_{j, i} \rho_{i} \sum_{i} \varepsilon_{j, i}\left(x_{i}^{\prime}-x_{i}\right)+\frac{1}{\kappa} \sum_{i} \varepsilon_{j, i} x_{i}^{\prime} \sum_{i} \delta_{j, i}\left(x_{i}^{\prime}-x_{i}\right)\right) \\
& =\frac{1}{\kappa} \sum\left(\sum_{i} \delta_{j, i} x_{i} \sum_{i} \varepsilon_{j, i}\left(x_{i}^{\prime}-x_{i}\right)+\sum_{i} \varepsilon_{j, i} x_{i}^{\prime} \sum_{i} \delta_{j, i}\left(x_{i}^{\prime}-x_{i}\right)\right) \\
& =\frac{1}{\kappa} \sum\left(-\sum_{i} \delta_{j, i} x_{i} \sum_{i} \varepsilon_{j, i} x_{i}+\sum_{i} \varepsilon_{j, i} x_{i}^{\prime} \sum_{i} \delta_{j, i} x_{i}^{\prime}\right)=-A
\end{aligned}
$$

\section{D.2 Proof of Claim 2}

Relying on the security of Waters signatures, we give an abstracted version of its proof, which in addition enables us to use some of its components in our proof: Unforgeability of Waters signatures is shown by specifying three algorithms SimPars, SimSig and Extract in order to employ an EUF-CMA-adversary $\mathcal{A}$ against a scheme for $m$-bit messages making $\ell$ signing queries to solve a CDH-instance $(X, Y)$ as follows:

$$
\begin{aligned}
& (s P a r, t r) \leftarrow \operatorname{SimPar}(X, Y, m, \ell) \\
& (M, \sigma) \leftarrow \mathcal{A}(s P a r, X: \operatorname{SimSig}(t r, \cdot)) \\
& Z \leftarrow \operatorname{Extract}(t r, M, \sigma)
\end{aligned}
$$

Some probability analysis then shows that if the adversary never queried $M$, the above experiment returns a CDH-solution with probability $\varepsilon^{\prime} \geq \frac{\varepsilon}{4 m \ell}$, where $\varepsilon$ is the adversary's advantage. In particular, the probability that all SimSig queries are answered correctly is $\frac{1}{2}$, whereas the probability that Extract actually transforms a valid forgery to a CDH-solution is $\frac{1}{2 m \ell}$.

Let $\mathcal{A}$ be an adversary against non-frameability of $\mathcal{D} \mathcal{S}$ making at most $\kappa$ PK-queries and at most $\ell$ Dlg and PSig (together) queries per user. We define a series of games, starting with the original experiment:

\section{Game 0: The Real Game}




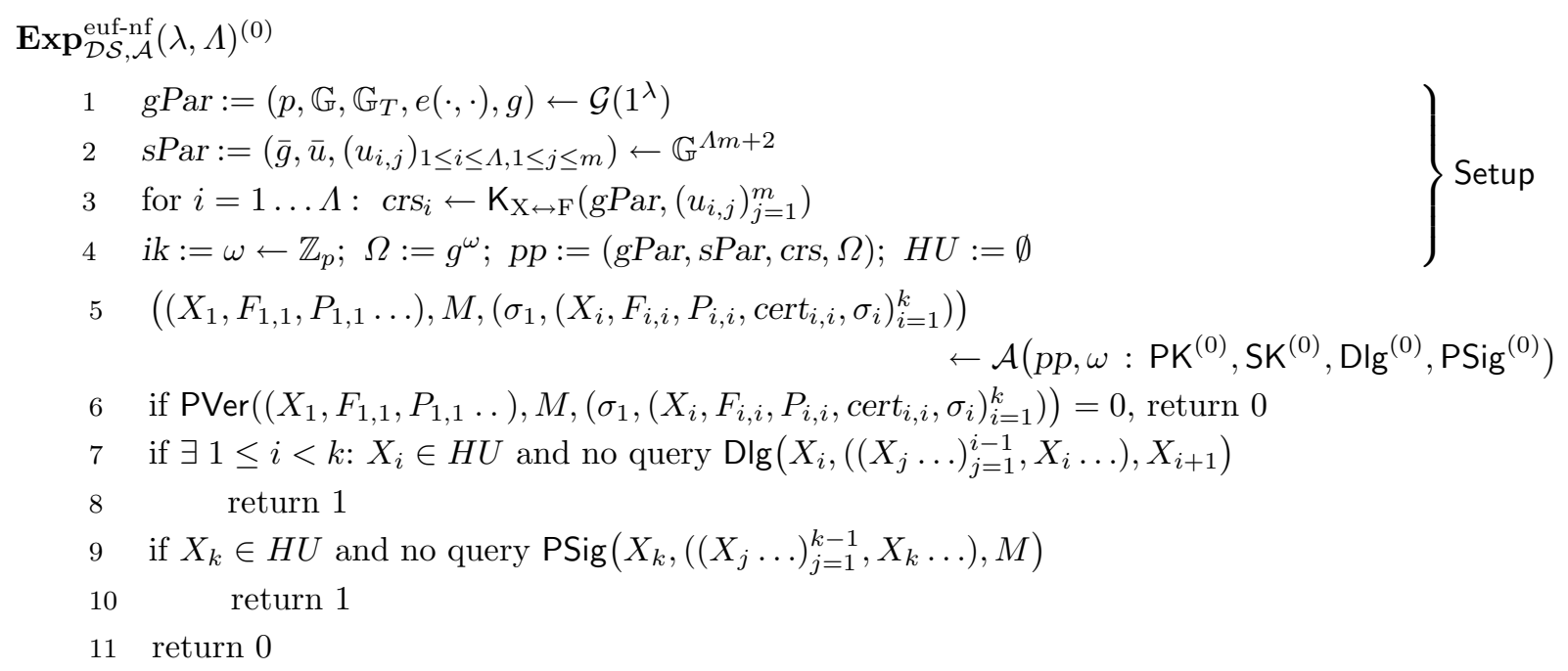

with the following oracles:

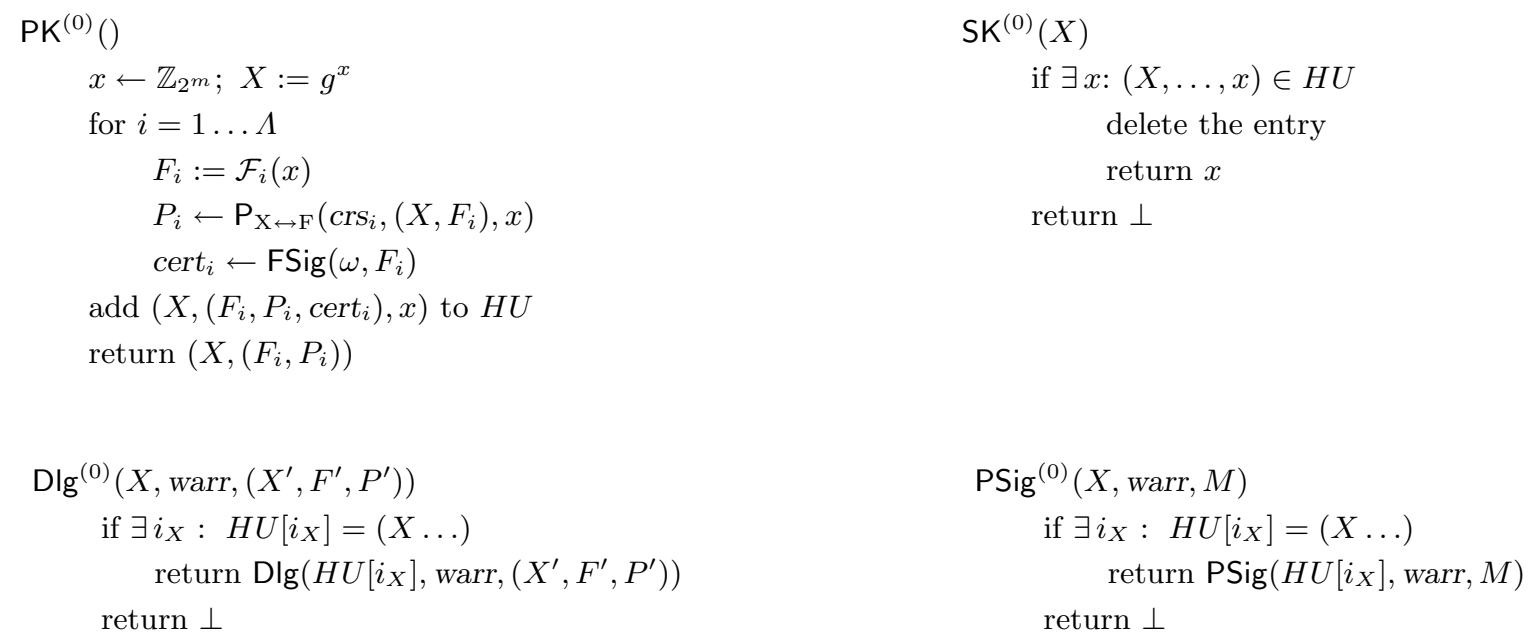

\section{Game 1: Choosing Target $X^{*}$ and Double-Checking the Proofs}




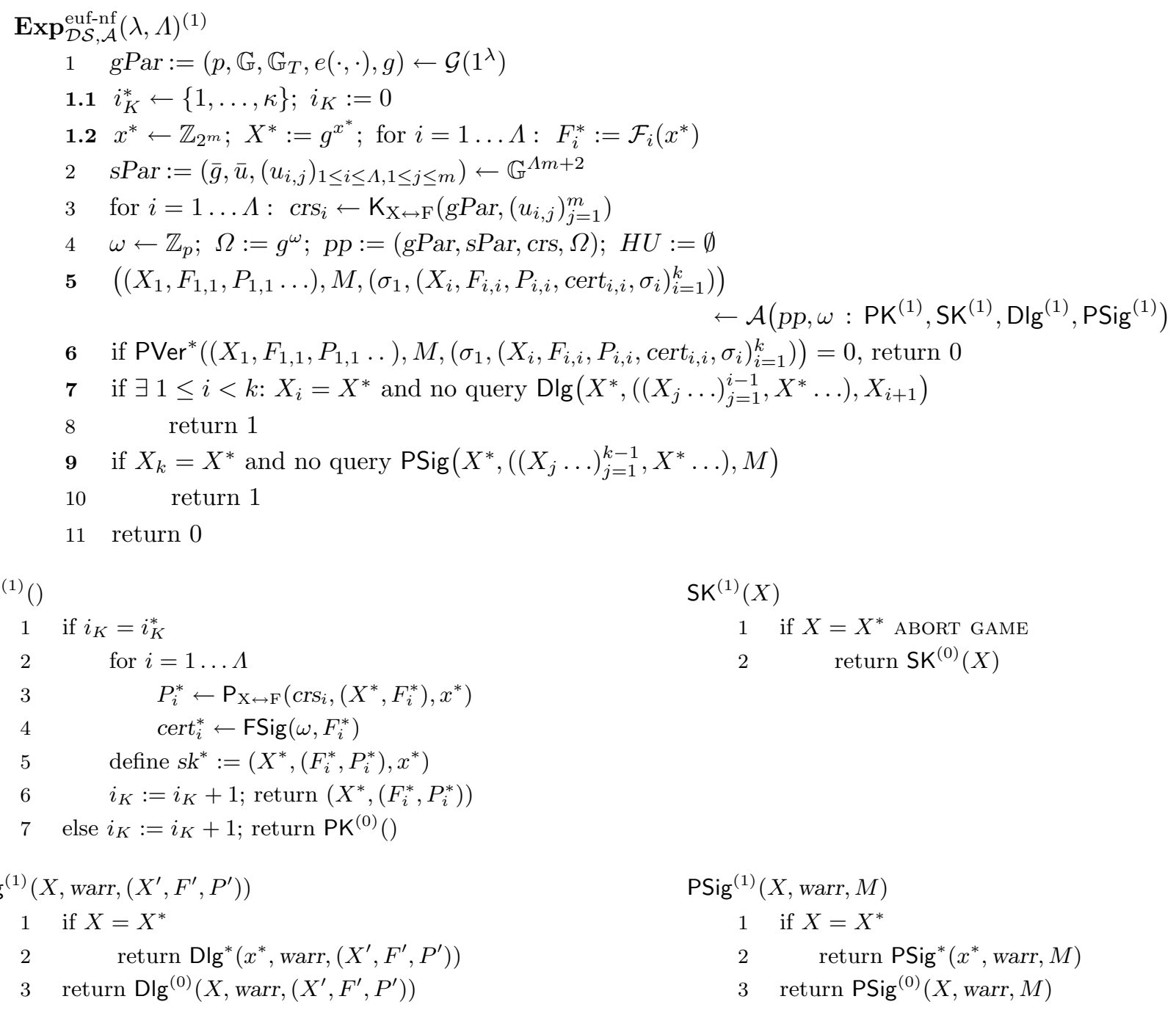

with PVer*, Dlg* and PSig* working as their non-starred version, except that $\mathrm{V}_{\mathrm{X} \leftrightarrow \mathrm{F}}$ is replaced by

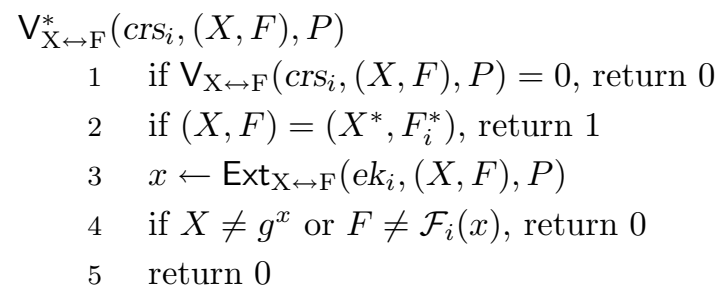

Game $0 \curvearrowright$ Game 1 First of all, soundness of $\Pi_{\mathrm{X} \leftrightarrow \mathrm{F}}$ ensures that replacing $\mathrm{V}_{\mathrm{X} \leftrightarrow \mathrm{F}}$ by $\mathrm{V}_{\mathrm{X} \leftrightarrow \mathrm{F}}$ only results in a negligible change, since a correct witness can be extracted from a valid proof with overwhelming probability. Second, since the choice of $i_{K}$ is random and independent of the rest of the game, an adversary winning Game 0 with probability $\varepsilon$ wins Game 1 with probability $\frac{1}{\kappa} \varepsilon$.

\section{Game 2: Simulating the Proofs for $X^{*}$}




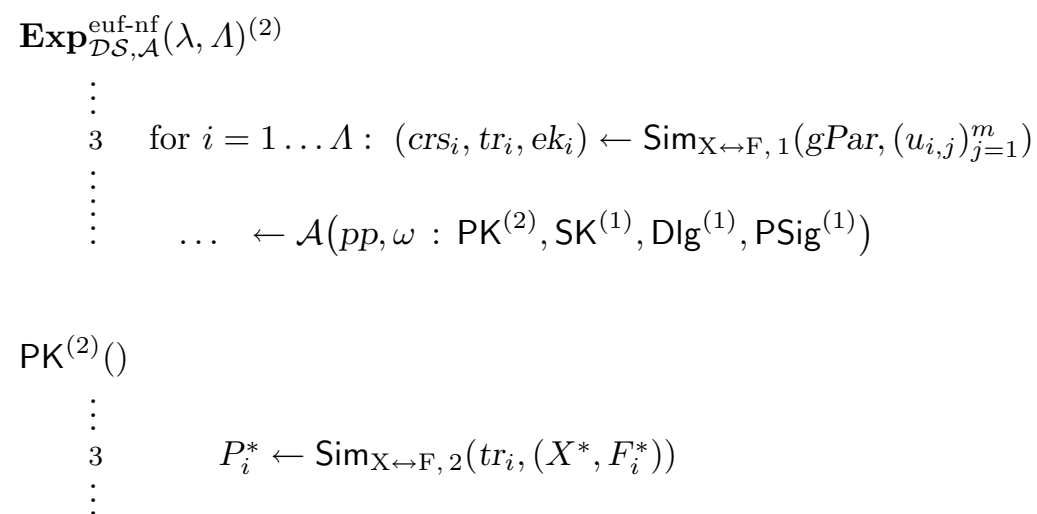

Game $1 \curvearrowright$ Game 2 Games $\operatorname{Exp}^{(1)}$ and $\operatorname{Exp}^{(2)}$ are indistinguishable by a hybrid argument on extraction zero knowledge of $\Pi_{\mathrm{X} \leftrightarrow \mathrm{F}}$. Consider hybrid game number $i$ : After receiving $c r s_{i}$, the distinguisher $\mathcal{D}$ simulates $\operatorname{Exp}^{(b)}$ using its extraction oracle for Line 3 of $\mathrm{V}_{\mathrm{X} \leftrightarrow \mathrm{F}}^{*}$. When eventually Line 3 of $\mathrm{PK}^{(b)}$ is reached, $\mathcal{D}$ outputs $\left(X^{*}, F_{i}^{*}\right)$ and receives $\mathrm{P}_{i}^{*}$ from the overlying experiment.

\section{Game 3: Simulating Signatures by $X^{*}$}

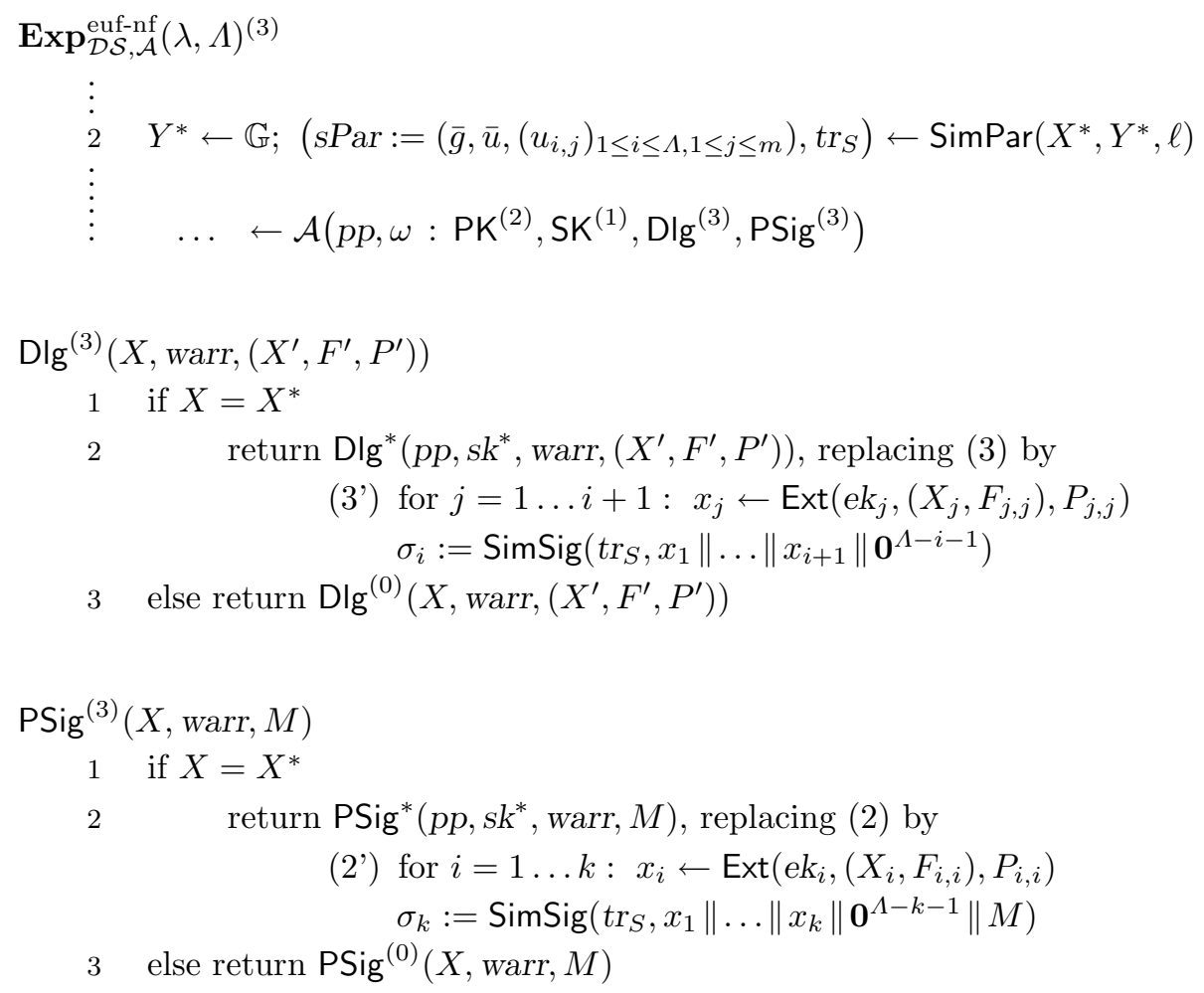

Game $2 \curvearrowright$ Game 3 First of all, note that due to the additional checks in PSig* introduced in Game 2, extracting and signing yields the same as signing the hash values directly. From the security proof for Waters signatures follows that the parameters created by SimPar are perfectly indistinguishable from actual ones and that the simulation is perfect at least half of the time. Since in addition, failure of simulation is independent from the adversary's view, an adversary winning Game 2 with probability $\varepsilon$ wins Game 3 with probability at least $\frac{1}{2} \varepsilon$. 


\section{Game 4: Beyond $\mathcal{L}_{\mathrm{X} \leftrightarrow \mathrm{F}}$}

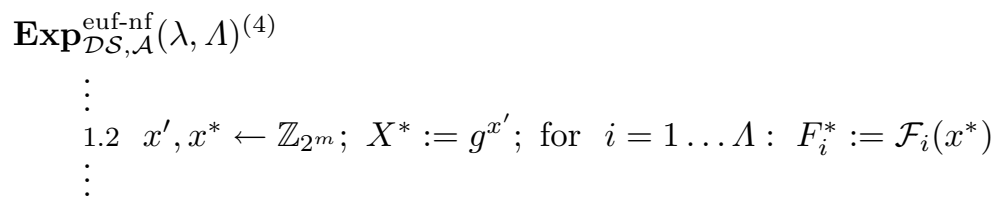

Game $3 \curvearrowright$ Game 4 Note that the private key $x^{*}$ in the original game is used to compute (1) the $F_{i}^{*}$, (2) the proofs $P_{i}^{*}$ and (3) the signatures $\sigma_{i}$. From Game 2 on, we compute $P_{i}^{*}$ without $x^{*}$ and from Game 3 on the signatures as well. Thus, a distinguisher between Games 3 and 4 yields a distinguisher for $\mathcal{L}_{\mathrm{X} \leftrightarrow \mathrm{F}}$, since after plugging in challenge $\left(X,\left(F_{i}\right)\right)$, the games can be simulated without knowledge of $x^{*}$.

The CDH-Adversary So far, we showed that any adversary having non-negligible advantage in winning Game 0 also wins Game 4 with non-negligible probability. If the experiment returns 1 in Line 8 then the fact that the signature returned by $\mathcal{A}$ passes PVer* implies that $\sigma_{i}$ is a signature on $F_{1,1} \cdots F_{i+1, i+1}$, thus actually a Waters signature of $x_{1}\|\ldots\| x_{i+1}$, the bits of the logarithms of $X_{1}, \ldots, X_{i+1}$. Now the condition in Line 7 guarantees that this very message has never been submitted to SimSig. (Note that verifying all proofs $P_{j, j}$ with $\mathrm{V}_{\mathrm{X} \leftrightarrow \mathrm{F}}^{*}$ ensures that the bits in $X_{j}$ and $F_{j, j}$ are the same.) An analogous argumentation holds if the experiment returns 1 in Line 10. Thus, to win the game $\mathcal{A}$ must in fact forge a Waters signature, which means we can use it to define a CDH-adversary by simulating Game 4 and instead of merely returning 1 extracting the bits from the proofs $P_{j, j}$ and feeding it to Extract.

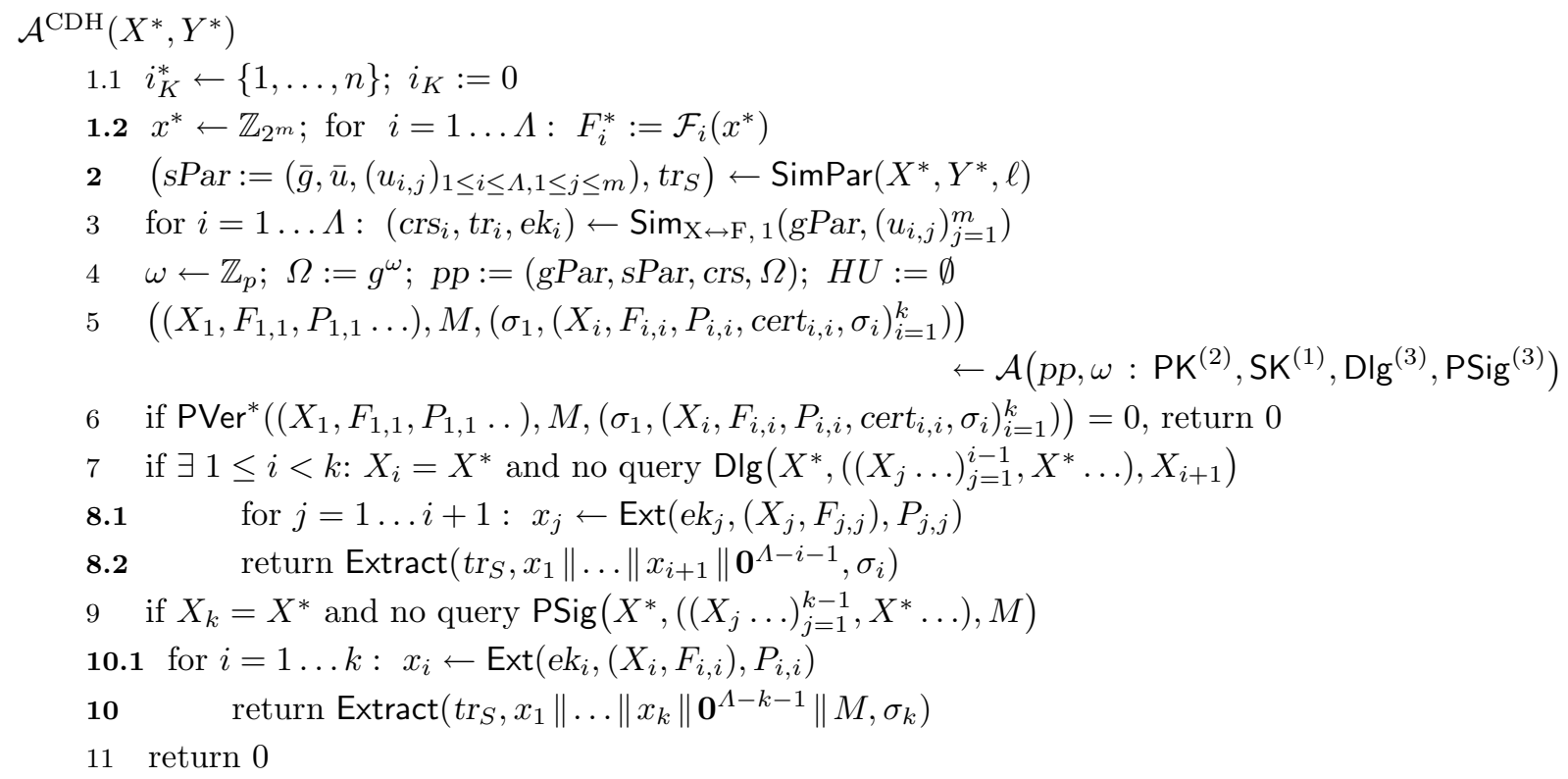

Game $4 \curvearrowright \mathbf{C D H}$ Game 4 is won if and only if the adversary manages to produce a Waters forgery. Now Waters' security proof guarantees that if all oracle queries were correctly simulated and the adversary returns a valid forgery then Extract computes a CDH solution from it with probability $\frac{1}{2 m \ell}$. It follows that if $\mathcal{A}$ wins Game 4 with probability $\varepsilon$, then $\mathcal{A}^{\mathrm{CDH}}$ produces a CDH-instance with probability at least $\frac{1}{2 m \ell} \varepsilon$.

All in all, we have thus shown that given an adversary $\mathcal{A}$ breaking non-frameability of $\mathcal{D S}$, we can construct an algorithm solving the CDH-problem with non-negligible probability. 